\title{
Index formulas and charge deficiencies on the Landau levels

\author{
Magnus Goffeng ${ }^{\text {a) }}$ \\ Department of Mathematical Sciences, Division of Mathematics, Chalmers University of \\ Technology and University of Gothenburg, Gothenburg 41296, Sweden
}

(Received 3 October 2009; accepted 30 November 2009; published online 2 February 2010)

\begin{abstract}
The notion of charge deficiency by Avron et al. ["Charge deficiency, charge transport and comparison of dimensions," Commun. Math. Phys. 159, 399 (1994)] is studied from the view of $K$-theory of operator algebras and is applied to the Landau levels in $\mathbb{R}^{2 n}$. We calculate the charge deficiencies at the higher Landau levels in $\mathbb{R}^{2 n}$ by means of an Atiyah-Singer-type index theorem. (C) 2010 American Institute of Physics. [doi:10.1063/1.3277159]
\end{abstract}

\section{INTRODUCTION}

This paper is a study of the charge deficiencies at the Landau levels in $\mathbb{R}^{2 n}$. The Landau levels are the eigenspaces of the Landau Hamiltonian which is the energy operator for a quantum particle moving in $\mathbb{R}^{2 n}$ under the influence of a constant magnetic field of full rank.

In Ref. 2, the notion of charge deficiency was introduced as a measure off how much a flux tube changes a fermionic system in $\mathbb{R}^{2}$. The setting of Ref. 2 is a quantum system where the Fermi energy is in a gap and the question is what happens when the system is taken through a cycle. Letting $P$ denote the projection onto the state space and $U$ the unitary transformation representing the cycle, the projection $Q$ onto the new state space after it had been taken through a cycle can be expressed as $Q=U P U^{*}$. The relative index $\operatorname{ind}(Q, P)$ is defined as an infinite dimensional analog of $\operatorname{dim} Q-\operatorname{dim} P$ and is well defined whenever $Q-P$ is a compact operator. The condition that $Q-P$ is compact is equivalent to that $[P, U]$ is compact. In the setting of Ref. 2 the relative index represents the change in the number of fermions that $U$ produces. In Ref. 2 the following formula was proven:

$$
\operatorname{ind}(Q, P)=\operatorname{ind}(P U P) \text {. }
$$

For sufficiently nice systems in $\mathbb{R}^{2}$ one can choose the particular unitary given by multiplication by the bounded function $U:=z /|z|$. The condition on the system that is needed is that $P$ commutes with $U$ up to a compact operator. The charge deficiency of a projection $P$ in the sense of Ref. 2 is then defined using $U$ as

$$
c(P):=\operatorname{ind}(P U P) .
$$

The viewpoint we will have in this paper is that the charge deficiency is a $K$-homology class. This viewpoint lies in line with the view on $D$-brane charges in string theory, see more in Refs. 6 and 14. In the case studied in Ref. 2 the charge deficiency is realized as an odd $K$-homology class on the circle $T$. The unitary $U$ defines a representation of $C(T)$ and using the fact that $P$ commutes with $U$ up to a compact operator we get a $K$-homology class. Let us denote this $K$-homology class by $[P]$ and by $u$ we will denote the generator of $C(\mathbb{T})$. In this notation, the charge deficiency is given by $c(P)=[P] \circ[u] \in K K(\mathrm{C}, \mathrm{C}) \cong \mathrm{Z}$, the Kasparov product between $[P] \in K^{1}(C(\mathbb{T}))$ and $[u] \in K_{1}(C(\mathbb{T}))$. Thus the charge deficiency is the image of $[P]$ under the isomorphism,

\footnotetext{
${ }^{a)}$ Electronic mail: goffeng@chalmers.se.
} 


$$
K^{1}\left(C\left(T^{\top}\right)\right)=K K_{1}(C(\mathbb{T}), \mathrm{C}) \cong \operatorname{Hom}\left(K_{1}\left(C\left(T^{\top}\right)\right), K_{0}(\mathrm{C})\right) \cong \mathbb{Z},
$$

where the first isomorphism is the natural mapping coming from the universal coefficient theorem for $K K$-theory and the second isomorphism comes from choosing $[u]$ as a generator for $K_{1}(C(T))$. So a better picture is that the $K$-homology class $[P] \in K^{1}(C(\mathbb{T}))$ is the charge deficiency of $P$.

The system we will consider in this paper consists of a particle moving in $\mathbb{R}^{2 n}$ under the influence of a constant magnetic field $B$ of full rank. If we choose a linear vector potential $A$ satisfying $\mathrm{d} A=B$ the Hamiltonian of this system is given by

$$
H_{A}:=(-i \nabla-A)^{2} .
$$

This Landau Hamiltonian should be viewed as a densely defined operator in the Hilbert space $L^{2}\left(\mathbb{R}^{2 n}\right)$. Taking $\mathcal{D}\left(H_{A}\right)=C_{c}^{\infty}\left(\mathbb{R}^{2 n}\right)$, the operator $H_{A}$ becomes essentially self-adjoint, see more in Ref. 12. Due to the identification $\mathbb{R}^{2 n}=C^{n}$ we will use the complex structure and we will assume that $B=\frac{i}{2} \sum \mathrm{d} z_{j} \wedge \mathrm{d} \bar{z}_{j}$.

The Landau Hamiltonian has a discrete spectrum with eigenvalues $\Lambda_{\ell}=2 \ell+n$ for $\ell \in \mathbb{N}$ and the eigenspaces $\mathcal{L}^{\ell}$ are infinite dimensional. Let

$$
P_{\ell}: L^{2}\left(\mathbb{R}^{2 n}\right) \rightarrow \mathcal{L}^{\ell}
$$

denote the orthogonal projection to the $\ell$ th eigenspace. Our point of view on the charge deficiencies for the Landau levels is that they are $K$-homology classes of the sphere $S^{2 n-1}$. For a bounded continuous function $a: \mathbb{R}^{2 n} \rightarrow M_{N}(\mathrm{C})$, we define the continuous function $a_{r} \in C\left(S^{2 n-1}\right)$ as

$$
a_{r}(v):=a(r v) .
$$

We let $A_{N}$ be the subalgebra of $C_{b}\left(\mathbb{R}^{2 n}\right) \otimes M_{N}(\mathrm{C})$, such that $a_{r}$ converges uniformly in $v$ to a continuous function $a_{\partial}$ on $S^{2 n-1}$. The mapping $a \mapsto a_{\partial}$ defines a $*$-homomorphism $A_{N}$ $\rightarrow C\left(S^{2 n-1}\right) \otimes M_{N}(\mathrm{C})$. The projection $P_{\ell}$ commutes up to a compact operator with $a \in A_{N}$ (see below in Theorem 3.2) and

$$
\left.P_{\ell} a\right|_{\mathcal{L}^{\ell} \otimes \mathrm{C}^{N}}: \mathcal{L}^{\ell} \otimes \mathrm{C}^{N} \rightarrow \mathcal{L}^{\ell} \otimes \mathrm{C}^{N}
$$

is Fredholm if and only if $a_{\partial}$ is invertible (see Proposition 3.6). Now we may present the main theorem of this paper.

Theorem 1: If $a_{\partial}$ is smooth and invertible, the index of $\left.P_{\ell} a\right|_{\mathcal{L}^{\ell} \otimes C^{N}}$ can be expressed as

$$
\operatorname{ind}\left(\left.P_{\ell} a\right|_{\mathcal{L}^{\ell} \otimes \mathrm{C}^{N}}\right)=\frac{-(\ell+n-1) !}{\ell !(2 n-1) !(2 \pi i)^{n}} \int_{S^{2 n-1}} \operatorname{tr}\left(\left(a_{\partial}^{-1} \mathrm{~d} a_{\partial}\right)^{2 n-1}\right) .
$$

The charge deficiency $\left[P_{\ell}\right] \in K^{1}\left(C\left(S^{2 n-1}\right)\right)$ may be expressed in terms of the Bergman projection $P_{B}$ on the unit ball in $\mathrm{C}^{n}$ as

$$
\left[P_{\ell}\right]=\frac{(\ell+n-1) !}{\ell !(n-1) !}\left[P_{B}\right]
$$

Following the advice of the referee, supported by the editor, we include an appendix containing some facts in $K$-theory needed for the exposition of this paper.

\section{THE PARTICULAR LANDAU LEVELS}

The spectral theory of the Landau Hamiltonian is well known and we will review it briefly. See more in Ref. 17. We will let $\varphi:=|z|^{2} / 4$ and assume that the magnetic field $B$ is of the form $B=i \partial \bar{\partial} \varphi$. Here $\partial$ is the complex linear part of the exterior differential d. Define the annihilation operators as 


$$
q_{j}:=2 \frac{\partial}{\partial \bar{z}_{j}}+z_{j} \text { for } j=1, \ldots, n .
$$

The adjoints are given by the creation operators $q_{j}^{*}:=-2\left(\partial / \partial z_{j}\right)+\bar{z}_{j}$. The annihilation and creation operators satisfy the following formulas:

$$
\left[q_{j}, q_{i}\right]=\left[q_{j}^{*}, q_{i}^{*}\right]=0, \quad\left[q_{i}, q_{j}^{*}\right]=2 \delta_{i j} \quad \text { and } \quad H_{A}=\sum_{j=1}^{n} q_{j}^{*} q_{j}+n=\sum_{j=1}^{n} q_{j} q_{j}^{*}-n
$$

Here we view $H_{A}$ as a densely defined operator in $L^{2}\left(\mathbb{C}_{1}^{n}\right)$. Thus the lowest eigenvalue is $n$ with corresponding eigenspace $\mathcal{L}_{0}=\mathrm{e}^{-\varphi} \mathcal{F}\left(\mathbb{C}^{n}\right)$, where $\mathcal{F}\left(\mathbb{C}^{n}\right):=L^{2}\left(\mathbb{C}^{n}, \mathrm{e}^{-2 \varphi}\right) \cap \mathcal{O}\left(\mathbb{C}^{n}\right)$ denotes the Fock space. Here $\mathcal{O}\left(\mathrm{C}^{n}\right)$ denotes the space of holomorphic functions in $\mathbb{C}^{n}$. In one complex dimension there is only one creation operator $q^{*}$ and the eigenspaces are given by $\mathcal{L}_{k}=\left(q^{*}\right)^{k} \mathcal{L}_{0}$. Using multi-index notation, for $\mathrm{k}=\left(k_{1}, \ldots, k_{n}\right) \in \mathbb{N}^{n}$, we define $q_{\mathrm{k}}:=q_{1}^{k_{1}} \cdots q_{n}^{k_{n}}$ and

$$
\mathcal{L}_{\mathrm{k}}:=q_{\mathrm{k}}^{*} \mathcal{L}_{0}=\mathcal{L}_{k_{1}} \otimes \mathcal{L}_{k_{2}} \otimes \cdots \otimes \mathcal{L}_{k_{n}}
$$

We will call this space for the particular Landau level of height $\mathrm{k}$. Using that $q_{j}$ and $q_{j}^{*}$ define a representation of the Heisenberg algebra in $n$ dimension, we obtain the eigenvalues of $H_{A}$ as $\Lambda_{\ell}=2 \ell+n$ with the corresponding eigenspaces,

$$
\mathcal{L}^{\ell}:=\underset{|\mathrm{k}|=\ell}{\oplus} \mathcal{L}_{\mathrm{k}}=\underset{|\mathrm{k}|=\ell}{\oplus} \mathcal{L}_{k_{1}} \otimes \mathcal{L}_{k_{2}} \otimes \cdots \otimes \mathcal{L}_{k_{n}}
$$

The $\ell$ th eigenspace $\mathcal{L}^{\ell}$ is called the Landau level of height $\ell$. Since the Hamiltonian commutes with the representation of $\mathrm{SU}(n)$ on $\mathrm{C}^{n}$, its eigenspaces are $\mathrm{SU}(n)$-invariant. Also the orthogonal projections $P_{\ell}: L^{2}\left(\mathbb{C}^{n}\right) \rightarrow \mathcal{L}^{\ell}$ are invariant under the $\mathrm{SU}(n)$-action.

Recall that the vacuum subspace $\mathcal{L}_{0} \subseteq L^{2}\left(\mathbb{C}^{n}\right)$ has a reproducing kernel induced by the reproducing kernel on the Fock space. The reproducing kernel of $\mathcal{F}\left(\mathrm{C}_{1}^{n}\right)$ is given by $K(z, w)=\mathrm{e}^{w \cdot \bar{z} / 4}$. So the reproducing kernel of $\mathcal{L}_{0}$ is given by

$$
K_{0}(z, w):=\mathrm{e}^{(1 / 4)\left(w \cdot \bar{z}-|z|^{2}-|w|^{2}\right)} .
$$

This expression for the reproducing kernel implies that the orthogonal projection $P_{0}: L^{2}\left(\mathbb{C}^{n}\right) \rightarrow \mathcal{L}_{0}$ is given by

$$
P_{0} f(z)=\int_{\mathbb{C}^{n}} f(w) \overline{K_{0}(z, w)} \mathrm{d} V
$$

By Ref. 16 the orthogonal projection $P_{\mathrm{k}}: L^{2}\left(\mathbb{C}^{n}\right) \rightarrow \mathcal{L}_{\mathrm{k}}$ onto the particular Landau level of height $\mathrm{k}$ is also an integral operator with kernel

$$
K_{\mathrm{k}}(z, w)=\mathrm{e}^{(1 / 4)\left(w \cdot \bar{z}-|z|^{2}-|w|^{2}\right)} \prod_{j=1}^{n} L_{k_{j}}\left(\frac{1}{2}\left|z_{j}-w_{j}\right|^{2}\right) .
$$

Here $L_{k}$ is the Laguerre polynomial of order $k$. Notice that the projections $P_{\mathrm{k}}$ are not $\mathrm{SU}(n)$-invariant, in general.

\section{TOEPLITZ OPERATORS ON THE LANDAU LEVELS}

We want to study topological properties of the particular Landau levels using Toeplitz operators. The symbols will be taken from a suitable subalgebra of $C_{b}\left(\mathrm{C}^{n}\right)$, the bounded functions on $\mathrm{C}^{n}$. The standard notation $\mathcal{B}(\mathcal{H})$ will be used for the bounded operators on a separable Hilbert space $\mathcal{H}$ and the compact operators will be denoted by $\mathcal{K}(\mathcal{H}) .{ }^{13}$ We will let $\pi: C_{b}\left(\mathbb{C}^{n}\right) \rightarrow \mathcal{B}\left(L^{2}\left(\mathbb{C}^{n}\right)\right)$ denote the representation given by pointwise multiplication. This is clearly an $\mathrm{SU}(n)$-equivariant mapping. Define the linear map $T_{\mathrm{k}}: C_{b}\left(\mathbb{C}^{n}\right) \rightarrow \mathcal{B}\left(\mathcal{L}_{\mathrm{k}}\right)$ by $T_{\mathrm{k}}(a):=\left.P_{\mathrm{k}} \pi(a)\right|_{\mathcal{L}_{\mathrm{k}}}$. 
Lemma 3.1: If $a \in C_{0}\left(\mathbb{C}^{n}\right)$ then $T_{\mathrm{k}}(a) \in \mathcal{K}\left(\mathcal{L}_{\mathrm{k}}\right)$ for all $\mathrm{k} \in \mathbb{N}^{n}$.

The proof of this lemma is analogous to the proof for the same statement for Toeplitz operators on a pseudoconvex domain from Ref. 18.

Proof: It is sufficient to prove the claim for $a \in C_{c}\left(\mathrm{C}^{n}\right)$, since $T_{\mathrm{k}}$ is continuous and $C_{c}\left(\mathrm{C}^{n}\right) \subseteq C_{0}\left(\mathrm{C}^{n}\right)$ is dense. Define the compact set $K:=\operatorname{supp}(a)$. Let $R: \mathcal{L}_{\mathrm{k}} \rightarrow L^{2}\left(\mathrm{C}^{n}\right)$ denote the operator given by multiplication by $\chi_{K}$, the characteristic function of $K$. We have $T_{\mathrm{k}}(a)$ $=P_{\mathrm{k}} \pi(a) R$ so the lemma holds if $R$ is compact. That $R$ is compact follows from Cauchy estimates of holomorphic functions on a compact set.

Define the $\mathrm{SU}(n)$-invariant $C^{*}$-subalgebra $A \subseteq C_{b}\left(\mathbb{C}^{n}\right)$ as consisting of functions $a$, such that $a(r v)$ converges uniformly in $v$ as $r \rightarrow \infty$ to a continuous function $a_{\partial}: S^{2 n-1} \rightarrow \mathrm{C}$ when $r \rightarrow \infty$. Thus we obtain a surjective $\mathrm{SU}(n)$-equivariant $*$-homomorphism $\pi_{\partial}: A \rightarrow C\left(S^{2 n-1}\right)$ given by

$$
\pi_{\partial}(a)(v):=\lim _{r \rightarrow \infty} a(r v) .
$$

The mapping $\pi_{\partial}$ satisfies ker $\pi_{\partial}=C_{0}\left(\mathrm{C}^{n}\right)$. We will henceforth consider $T_{\mathrm{k}}$ as a mapping from $A$ to $\mathcal{B}\left(\mathcal{L}_{\mathrm{k}}\right)$.

If we let $B_{n}$ denote the open unit ball in $\mathrm{C}^{n}$, another view on $A$ is as the image of the $\mathrm{SU}(n)$-equivariant $*$-monomorphism $C\left(\overline{B_{n}}\right) \rightarrow C_{b}\left(B_{n}\right) \cong C_{b}\left(\mathrm{C}^{n}\right)$, where the last isomorphism comes from an $\mathrm{SU}(n)$-equivariant homeomorphism $B_{n} \cong \mathrm{C}^{n}$.

Theorem 3.2: The projection $P_{\mathrm{k}}$ satisfies $\left[P_{\mathrm{k}}, \pi(a)\right] \in \mathcal{K}\left(L^{2}\left(\mathrm{C}^{n}\right)\right)$ for all $a \in A$. Therefore, the *-linear mapping $T_{\mathrm{k}}: A \rightarrow \mathcal{B}\left(\mathcal{L}_{\mathrm{k}}\right)$ satisfies

$$
T_{\mathrm{k}}(a b)-T_{\mathrm{k}}(a) T_{\mathrm{k}}(b) \in \mathcal{K}\left(\mathcal{L}_{\mathrm{k}}\right) .
$$

The proof is based on a similar result from Ref. 3 where the Fock space was used to define a Toeplitz quantization of a certain subalgebra of $L^{\infty}\left(\mathrm{C}^{n}\right)$. The case of the Fock space is more or less the same as the case $\mathrm{k}=0$ for Landau quantization. To prove the theorem we need a lemma similar to part (iv) of Theorem 5 of Ref. 3. Using the isomorphism $A \cong C\left(\overline{B_{n}}\right)$ we define the dense subalgebra $A_{1} \subseteq A$ as the inverse image of the Lipschitz continuous functions in $C\left(\overline{B_{n}}\right)$.

Lemma 3.3: For $a \in A_{1}$ then for any $\varepsilon>0$ we may write $a=g_{\varepsilon}+h_{\varepsilon}$ where $h_{\varepsilon} \in C_{0}\left(\mathrm{C}^{n}\right)$ and $g_{\varepsilon} \in A$ satisfies

$$
\left|g_{\varepsilon}(z)-g_{\varepsilon}(w)\right| \leq \varepsilon|z-w| \quad \forall z, w \in \mathbb{C}^{n} .
$$

Proof: Let $C$ denote the Lipschitz constant of $\pi_{\partial}(a)$. Take an $\varepsilon>0$ and let $\chi_{\varepsilon}$ be a Lipshitz continuous $\mathrm{SU}(n)$-invariant cutoff, such that $\chi_{\varepsilon}(z)=0$ for $|z| \leq R$ and $\chi_{\varepsilon}(z)=0$ for $|z| \geq 2 R$, where $R=R(\varepsilon, C)$ is to be defined later. To shorten notation, define $a_{\partial}:=\pi_{\partial}(a)$. Let

$$
g_{\varepsilon}(z):=\chi_{\varepsilon}(z) \cdot a_{\partial}(z /|z|)
$$

and $h_{\varepsilon}:=a-g_{\varepsilon}$. Clearly $h_{\varepsilon} \in C_{0}\left(C^{n}\right)$ and $g_{\varepsilon} \in A$ so what remains to be proven is that $R$ can be chosen in such a way that $g_{\varepsilon}$ satisfies Eq. (2).

We have elementary estimates

$$
\left|\frac{z}{|z|}-\frac{w}{|w|}\right| \leq \frac{|z-w|}{|z|}+\left|\frac{w}{|z|}-\frac{w}{|w|}\right| \leq 2 \frac{|z-w|}{|w|} .
$$

Thus, for $z, w \neq 0$ the function $a_{\partial}$ satisfies

$$
\left|a_{\partial}\left(\frac{z}{|z|}\right)-a_{\partial}\left(\frac{w}{|w|}\right)\right| \leq \frac{2 C}{|w|}|z-w| .
$$

The function $\chi_{\varepsilon}$ has Lipschitz coefficient $1 / R$ so if we take $R>2 C / \varepsilon$ then $g_{\varepsilon}$ satisfies Eq. (2)

Let $\mathcal{C}\left(L^{2}\left(C^{n}\right)\right):=\mathcal{B}\left(L^{2}\left(C^{n}\right)\right) / \mathcal{K}\left(L^{2}\left(\mathrm{C}^{n}\right)\right)$ denote the Calkin algebra and $\mathfrak{q}$ the quotient mapping.

Proof of Theorem 3.2: Since Lipschitz continuous functions are dense in $A$ we may assume 
that $a \in A_{1}$, so by Lemma 3.3 we can for any $\varepsilon>0$ write $a=g_{\varepsilon}+h_{\varepsilon}$. In this case we have for $f$ $\in L^{2}\left(C^{n}\right)$

$$
\left[P_{\mathrm{k}}, \pi\left(g_{\varepsilon}\right)\right] f(z)=\int\left(g_{\varepsilon}(z)-g_{\varepsilon}(w)\right) K_{\mathrm{k}}(z, w) f(w) \mathrm{d} w .
$$

Define the operator

$$
B f(z):=\int|z-w| K_{\mathrm{k}}(z, w) f(w) \mathrm{d} w .
$$

By Eq. (1) we have that for some $C$ the integral kernel of $B$ is bounded by

$$
|z-w|\left|K_{\mathrm{k}}(z, w)\right| \leq C|z-w||\mathrm{k}|+1 \mathrm{e}^{-(1 / 8)|z-w|^{2}} .
$$

Therefore, the kernel of $B$ is dominated by the kernel of a bounded convolution operator and $\|B\|<\infty$. The estimate (2) for $g_{\varepsilon}$ implies that

$$
\left\|\left[P_{\mathrm{k}}, \pi\left(g_{\varepsilon}\right)\right]\right\| \leq \varepsilon\|B\| .
$$

Using that $\left[P_{\mathrm{k}}, \pi\left(g_{\varepsilon}\right)\right]=\left[P_{\mathrm{k}}, \pi(a)\right]$ modulo compact operators, by Lemma 3.1, we have the inequality

$$
\left\|\mathfrak{q}\left(\left[P_{\mathrm{k}}, a\right]\right)\right\|_{\mathcal{C}\left(L^{2}\left(\mathrm{C}^{n}\right)\right)} \leq \varepsilon\|B\| \quad \forall \varepsilon>0 .
$$

Therefore, $\mathfrak{q}\left(\left[P_{\mathrm{k}}, a\right]\right)=0$ and $\left[P_{\mathrm{k}}, a\right]$ is compact.

Theorem 3.2 implies that the mapping $\tilde{\beta}_{\mathrm{k}}:=\mathfrak{q} \circ T_{\mathrm{k}}: A \rightarrow \mathcal{C}\left(\mathcal{L}_{\mathrm{k}}\right)$ is a well defined *-homomorphism. Define the $C^{*}$-algebra

$$
\widetilde{\mathcal{T}}_{\mathrm{k}}:=\left\{a \oplus x \in A \oplus \mathcal{B}\left(\mathcal{L}_{\mathrm{k}}\right): \widetilde{\beta}_{\mathrm{k}}(a)=\mathfrak{q}(x)\right\} .
$$

This $C^{*}$-algebra contains $\mathcal{K}$ as an ideal via the embedding $k \mapsto 0 \oplus k$ and we obtain a short exact sequence,

$$
0 \rightarrow \mathcal{K} \rightarrow \widetilde{\mathcal{T}}_{\mathrm{k}} \rightarrow A \rightarrow 0
$$

Lemma 3.4: Let $\left(\mathrm{k}_{p}\right)_{p=1}^{N} \subseteq \mathbb{N}^{n}$ be a finite collection of distinct $n$-tuples of integers. Then the mapping

$$
A \ni a \mapsto \mathfrak{q}\left(\left(\sum_{p=1}^{N} P_{\mathrm{k}_{p}}\right) \pi(a)\left(\sum_{p=1}^{N} P_{\mathrm{k}_{p}}\right)\right) \in \mathcal{C}\left(\oplus_{p=1}^{N} \mathcal{L}_{\mathrm{k}_{p}}\right)
$$

coincides with the mapping

$$
A \ni a \mapsto \oplus_{p=1}^{N} \widetilde{\beta}_{\mathrm{k}_{p}}(a) \in \mathcal{C}\left(\oplus_{p=1}^{N} \mathcal{L}_{\mathrm{k}_{p}}\right) .
$$

Proof: The lemma follows if we show that $P_{\mathrm{k}} \pi(a) P_{\mathrm{k}^{\prime}} \in \mathcal{K}\left(L^{2}\left(\mathrm{C}^{n}\right)\right)$ for $\mathrm{k} \neq \mathrm{k}^{\prime}$. But Theorem 3.2 implies that $P_{\mathrm{k}} \pi(a)\left(1-P_{\mathrm{k}}\right) \in \mathcal{K}\left(L^{2}\left(\mathrm{C}^{n}\right)\right)$. So the lemma follows from

$$
P_{\mathrm{k}} \pi(a) P_{\mathrm{k}^{\prime}}=P_{\mathrm{k}} \pi(a)\left(1-P_{\mathrm{k}}\right) P_{\mathrm{k}^{\prime}} .
$$

In particular, we can look at the collection of all $\mathrm{k}: \mathrm{s}$, such that $|\mathrm{k}|=\ell$. We will define the $\mathrm{SU}(n)$-equivariant mapping $\widetilde{\beta}_{\ell}: A \rightarrow \mathcal{C}\left(\mathcal{L}^{\ell}\right)$ as 


$$
a \mapsto \oplus_{|\mathrm{k}|=\ell} \widetilde{\beta}_{\mathrm{k}}(a) .
$$

Just as for the particular Landau levels we define

$$
\tilde{\mathcal{T}}^{\ell}:=\left\{a \oplus x \in A \oplus \mathcal{B}\left(\mathcal{L}^{\ell}\right): \widetilde{\beta}_{\ell}(a)=\mathfrak{q}(x)\right\} .
$$

The projection map $\tilde{\mathcal{T}}^{\ell} \rightarrow A$ given by $a \oplus x \mapsto a$ defines an $\mathrm{SU}(n)$-equivariant extension,

$$
0 \rightarrow \mathcal{K} \rightarrow \tilde{\mathcal{T}}^{\ell} \rightarrow A \rightarrow 0
$$

Lemma 3.5: The kernel of $\widetilde{\beta}_{\ell}$ is $C_{0}\left(\mathrm{C}^{n}\right)$.

Proof: Lemma 3.1 implies that $C_{0}\left(\mathbb{C}^{n}\right) \subseteq$ ker $\widetilde{\beta}_{\ell}$. To prove the reverse inclusion we observe that the mapping $\widetilde{\beta}_{\ell}$ is a unital $\mathrm{SU}(n)$-equivariant $*$-homomorphism. Since $\widetilde{\beta}_{\ell}$ is equivariant, the ideal ker $\widetilde{\beta}_{\ell} \subseteq A$ is $\mathrm{SU}(n)$-invariant. The inclusion $C_{0}\left(\mathrm{C}^{n}\right) \subseteq \operatorname{ker} \widetilde{\beta}_{\mathrm{k}}$ implies that there is an equivariant surjection $C\left(S^{2 n-1}\right) \rightarrow A / \operatorname{ker} \widetilde{\beta}_{\ell}$ which must be an isomorphism since $C\left(S^{2 n-1}\right)$ is $\mathrm{SU}(n)$-simple and $\widetilde{\beta}_{\ell}$ is unital. It follows that $\operatorname{ker} \widetilde{\beta}_{\ell}=C_{0}\left(\mathrm{C}^{n}\right)$.

It is interesting that although the statement of Lemma 3.5 sounds algebraic, it is really the analytic statement that $T_{\ell}(a)$ is compact if and only if $a$ vanishes at infinity. Moreover, this is proven with algebraic methods!

Proposition 3.6: If $u \in A \otimes M_{N}$, the operator $T_{\ell}(u)$ is Fredholm if and only if $\pi_{\partial}(u)$ is invertible.

Proof: By Atkinson's theorem $T_{\ell}(u)$ is Fredholm if and only if $\widetilde{\beta}_{\ell}(u)$ is invertible. Lemma 3.5 implies that $\operatorname{ker} \pi_{\partial}=\operatorname{ker} \widetilde{\beta}_{\ell}$ so $\widetilde{\beta}_{\ell}(u)$ is invertible if and only if $\pi_{\partial}(u)$ is invertible.

\section{PULLING SYMBOLS BACK FROM $S^{2 n-1}$}

To put the Toeplitz operators on a Landau level in a suitable homological picture, we must pass from $A$ to $C\left(S^{2 n-1}\right)$. This is a consequence of the circumstance that $A$ is homotopy equivalent to $\mathrm{C}$, so $A$ does not contain any relevant topological information. With Lemma 3.5 in mind we define the Toeplitz algebra $\mathcal{T}_{\mathrm{k}}$ for $C\left(S^{2 n-1}\right)$ as if $\beta_{\mathrm{k}}$ were injective. So let $\lambda: C\left(S^{2 n-1}\right) \rightarrow \mathcal{B}\left(L^{2}\left(C^{n}\right)\right)$ denote the *-representation defined by

$$
\lambda(a) f(z)=a\left(\frac{z}{|z|}\right) f(z) .
$$

Take $\chi_{0} \in C^{\infty}(\mathrm{R})$ to be a smooth function such that $\chi_{0}(x)=0$ for $|x| \leq 1$ and $1-\chi_{0} \in C_{c}^{\infty}(\mathbb{R})$. We define the cutoff $\chi(z):=\chi_{0}(|z|)$ and the operator

$$
\widetilde{P}_{\mathrm{k}}:=P_{\mathrm{k}} \chi .
$$

For the operator $\widetilde{P}_{\mathrm{k}}, \mathfrak{q}\left(\widetilde{P}_{\mathrm{k}}\right)$ is a projection by Lemma 3.1. We let $\mathcal{T}_{\mathrm{k}}$ be the $C^{*}$-algebra generated by $\widetilde{P}_{\mathrm{k}} \lambda\left(C\left(S^{2 n-1}\right)\right) \widetilde{P}_{\mathrm{k}}^{*}$.

Theorem 4.1: For any $\mathrm{k}, \mathrm{k}^{\prime} \in \mathbb{N}^{n}$ there exist a unitary

$$
Q_{\mathrm{k}, \mathrm{k}^{\prime}}: \mathcal{L}_{\mathrm{k}^{\prime}} \rightarrow \mathcal{L}_{\mathrm{k}},
$$

such that $\operatorname{Ad}\left(Q_{\mathrm{k}, \mathrm{k}^{\prime}}\right): \mathcal{T}_{\mathrm{k}} \rightarrow \mathcal{T}_{\mathrm{k}^{\prime}}$ is an isomorphism satisfying

$$
\mathfrak{q}\left(\widetilde{P}_{\mathrm{k}^{\prime}} \lambda(a) \widetilde{P}_{\mathrm{k}^{\prime}}^{*}\right)=\mathfrak{q} \circ \operatorname{Ad}\left(Q_{\mathrm{k}, \mathrm{k}^{\prime}}\right)\left(\widetilde{P}_{\mathrm{k}} \lambda(a) \widetilde{P}_{\mathrm{k}}^{*}\right) .
$$

Furthermore, for any $\mathrm{k} \in \mathbb{N}^{n}$, the representation of $\mathcal{T}_{\mathrm{k}}$ on $\mathcal{L}_{\mathrm{k}}$ given by the inclusion $\mathcal{T}_{\mathrm{k}} \subseteq \mathcal{B}\left(\mathcal{L}_{\mathrm{k}}\right)$ is irreducible and has the cyclic vector $\xi_{\mathrm{k}}$ defined by

$$
\xi_{\mathrm{k}}(z):=q_{\mathrm{k}}^{*}\left(\mathrm{e}^{-|z|^{2} / 4}\right) .
$$


Up to normalization the cyclic vectors satisfy

$$
Q_{\mathrm{k}, \mathrm{k}^{\prime}} \xi_{\mathrm{k}^{\prime}}=\xi_{\mathrm{k}} .
$$

Proof: Let us start with observing that for any $a, b \in C\left(S^{2 n-1}\right)$ we have

$$
\widetilde{P}_{\mathrm{k}} \lambda(a b) \widetilde{P}_{\mathrm{k}}^{*}-\widetilde{P}_{\mathrm{k}} \lambda(a) \widetilde{P}_{\mathrm{k}}^{*} P_{\mathrm{k}} \lambda(b) \widetilde{P}_{\mathrm{k}}^{*} \in \mathcal{K} .
$$

So if $\mathcal{T}_{\mathrm{k}}$ acts irreducibly on $\mathcal{L}_{\mathrm{k}}$, then $\mathcal{K} \subseteq \mathcal{T}_{\mathrm{k}}$.

First we will construct a cyclic vector for the $\mathcal{T}_{\mathrm{k}}$-action on $\mathcal{L}_{\mathrm{k}}$ and use the cyclic vector in $\mathcal{L}_{0}$ to show that $\mathcal{T}_{0}$ acts irreducibly on $\mathcal{L}_{0}$. Then we will show that for k such that $\mathcal{T}_{\mathrm{k}}$ acts irreducibly on $\mathcal{L}_{\mathrm{k}}$ and $1 \leq j \leq n$ there is an isomorphism $\mathcal{T}_{\mathrm{k}} \cong \mathcal{T}_{\mathrm{k}+e_{j}}$ induced by a unitary intertwining the $\mathcal{T}_{\mathrm{k}}$-action on $L_{\mathrm{k}}$ with the $\mathcal{T}_{\mathrm{k}+e_{j}}$-action on $\mathcal{L}_{\mathrm{k}+e_{j}}$.

Consider the elements $\xi_{\mathrm{m}, \mathrm{k}} \in \mathcal{L}_{\mathrm{k}}$ for $\mathrm{m} \in \mathbb{N}^{n}$ defined by

$$
\xi_{\mathrm{m}, \mathrm{k}}(z):=q_{\mathrm{k}}^{*}\left(z^{\mathrm{m}} \mathrm{e}^{-|z|^{2} / 4}\right) .
$$

The elements $\xi_{\mathrm{m}, \mathrm{k}}$ form an orthogonal basis for $\mathcal{L}_{\mathrm{k}}$. As in the statement of the theorem, we define $\xi_{\mathrm{k}}:=\xi_{0, \mathrm{k}}$. For $a \in C\left(S^{2 n-1}\right)$ we have

$$
\left\langle\xi_{\mathrm{m}, \mathrm{k}}, \widetilde{P}_{\mathrm{k}} a \widetilde{P}_{\mathrm{k}}^{*} \xi_{\mathrm{k}}\right\rangle=\left\langle\xi_{\mathrm{m}, \mathrm{k}}, \chi^{2} a \xi_{\mathrm{k}}\right\rangle=\int_{\mathbb{C}^{n}} \bar{q}_{\mathrm{k}}^{*}\left(\bar{z}^{\mathrm{m}} \mathrm{e}^{-|z|^{2} / 4}\right) q_{\mathrm{k}}^{*}\left(\mathrm{e}^{-|z|^{2} / 4}\right) \chi^{2}(z) a\left(\frac{z}{|z|}\right) \mathrm{d} V=\int_{S^{2 n-1}} p_{\mathrm{m}}(\bar{z}) a(z) \mathrm{d} S
$$

for some polynomials $p_{\mathrm{m}}$ of degree at most $2|\mathrm{k}|+|\mathrm{m}|$. It follows that $\mathcal{T}_{\mathrm{k}} \xi_{\mathrm{k}}$ span $\mathcal{L}_{\mathrm{k}}$ and therefore $\overline{\mathcal{T}_{\mathrm{k}} \xi_{\mathrm{k}}}=\mathcal{L}_{\mathrm{k}}$. Thus, $\xi_{\mathrm{k}}$ is a cyclic vector for the $\mathcal{T}_{\mathrm{k}}$-action.

By standard theory $\mathcal{T}_{0}$ acts irreducibly on $\mathcal{L}_{0}$ if and only if there are no nonzero $\xi_{0}^{\prime}, \xi_{0}^{\prime \prime} \in \mathcal{L}_{0}$, such that $\xi_{0}=\xi_{0}^{\prime}+\xi_{0}^{\prime \prime}$ and $\mathcal{T}_{0} \xi_{0}^{\prime} \perp \mathcal{T}_{0} \xi_{0}^{\prime \prime}$. Assume that for some $\xi_{0}^{\prime} \in \mathcal{L}_{0}$ we have $\mathcal{T}_{0} \xi_{0}^{\prime} \perp \mathcal{T}_{0}\left(\xi_{0}-\xi_{0}^{\prime}\right)$. The orthogonality condition implies that $\left\langle\widetilde{P}_{0} a \widetilde{P}_{0}^{*}\left(\xi_{0}-\xi_{0}^{\prime}\right), \xi_{0}^{\prime}\right\rangle=0$ for all $a \in C\left(S^{2 n-1}\right)$ and $P_{0}$ is self-adjoint so this relation is equivalent to $\left\langle\chi^{2} a \xi_{0}, \xi_{0}^{\prime}\right\rangle=\left\langle\chi^{2} a \xi_{0}^{\prime}, \xi_{0}^{\prime}\right\rangle$ for all $a \in C\left(S^{2 n-1}\right)$. There exist a holomorphic function $f_{0}$, such that $\xi_{0}^{\prime}(z)=f_{0}(z) \mathrm{e}^{-|z|^{2} / 4}$ and the equation $\left\langle\chi^{2} a \xi_{0}, \xi_{0}^{\prime}\right\rangle=\left\langle\chi^{2} a \xi_{0}^{\prime}, \xi_{0}^{\prime}\right\rangle$ implies

$$
\int_{\mathbb{C}^{n}} \overline{f_{0}(z)} \mathrm{e}^{-|z|^{2} / 2} \chi^{2}(z) a\left(\frac{z}{|z|}\right) \mathrm{d} V=\int_{\mathbb{C}^{n}}\left|f_{0}(z)\right|^{2} \mathrm{e}^{-|z|^{2} / 2} \chi^{2}(z) a\left(\frac{z}{|z|}\right) \mathrm{d} V
$$

Hence $f_{0}$ must be real, and since it is holomorphic it must be constant. Thus $\xi_{0}^{\prime}$ is in the linear span of $\xi_{0}$ and $\xi_{0}$ defines a pure state. Since the $\mathcal{T}_{0}$-action on $\mathcal{L}_{0}$ has a pure state, it is irreducible.

Assume that $\mathcal{T}_{\mathrm{k}}$ acts irreducibly on $\mathcal{L}_{\mathrm{k}}$. Consider the polar decomposition of the unbounded operator $q_{j}$ on $L^{2}\left(\mathrm{C}^{n}\right)$, that is, $q_{j}^{*}=E_{j} Q_{j}$, where $Q_{j}$ is a coisometry and $E_{j}$ is a strictly positive unbounded operator. Clearly $E_{j}$ is diagonal on the energy levels and

$$
E_{j}=\underset{\mathrm{k}^{\prime} \in \mathbb{N}^{n}}{\oplus} \sqrt{k_{j}^{\prime}} P_{\mathrm{k}^{\prime}} .
$$

We define the $*$-homomorphism $\rho_{j}: \mathcal{T}_{\mathrm{k}+e_{j}} \rightarrow \mathcal{B}\left(\mathcal{L}_{\mathrm{k}}\right)$ by $\rho_{j}(T):=\left.Q_{j}^{*} T Q_{j}\right|_{\mathcal{L}_{\mathrm{k}}}$. Since $Q_{j}$ is a coisometry this is clearly a $*$-monomorphism. It follows from the fact that $q_{j}^{*} \mid: \mathcal{L}_{\mathrm{k}} \rightarrow \mathcal{L}_{\mathrm{k}+e_{j}}$ is an isomorphism, that $Q_{j} \mid: \mathcal{L}_{\mathrm{k}} \rightarrow \mathcal{L}_{\mathrm{k}+e_{j}}$ is unitary, so $\rho_{j}$ is unital. If $a \in C^{\infty}\left(S^{2 n-1}\right)$ then for some nonzero constant $c$ we have

$$
\rho_{j}\left(\widetilde{P}_{\mathrm{k}+e_{j}} \lambda(a) \widetilde{P}_{\mathrm{k}+e_{j}}^{*}\right)=\left.c q_{j} \widetilde{P}_{\mathrm{k}+e_{j}} \lambda(a) \widetilde{P}_{\mathrm{k}+e_{j}}^{*} q_{j}^{*}\right|_{\mathcal{L}_{\mathrm{k}}}=\left.c P_{\mathrm{k}}\left[\frac{\partial}{\partial \bar{z}_{j}}, \chi^{2} \lambda(a)\right] P_{\mathrm{k}+e_{j}} q_{j}^{*}\right|_{\mathcal{L}_{\mathrm{k}}}+\widetilde{P}_{\mathrm{k}} \lambda(a) \widetilde{P}_{k}^{*} \in \mathcal{T}_{\mathrm{k}}
$$

because Theorem 3.2 implies $P_{\mathrm{k}} b P_{\mathrm{k}+e_{j}} \in \mathcal{K}\left(L^{2}\left(\mathrm{C}^{n}\right)\right)$ for $b \in A$ and by the induction assumption $\mathcal{K} \subseteq \mathcal{T}_{\mathrm{k}}$. So we obtain a $*$-monomorphism $\rho_{j}: \mathcal{T}_{\mathrm{k}+e_{j}} \rightarrow \mathcal{T}_{\mathrm{k}}$. However, we have cyclic vectors $\xi_{\mathrm{k}}$ and $\xi_{\mathrm{k}+e_{j}}$ for $\mathcal{T}_{\mathrm{k}}$, respectively, $\mathcal{T}_{\mathrm{k}+e_{j}}$. For these vectors, $Q_{j} \xi_{\mathrm{k}}$ is a multiple of $\xi_{\mathrm{k}+e_{j}}$ so 


$$
\mathcal{L}_{\mathrm{k}+e_{j}}=\overline{\mathcal{T}}_{{\mathrm{k}+e_{j}}_{\xi_{\mathrm{k}+e_{j}}}}^{Q_{j}^{*}} \overline{\mathcal{T}_{\mathrm{k}} \xi_{\mathrm{k}}} .
$$

Therefore, $\rho_{j}$ is surjective and an isomorphism. We conclude that $\mathcal{T}_{\mathrm{k}}$ is independent of $\mathrm{k}$ and the representations on $\mathcal{L}_{\mathrm{k}}$ are irreducible since $\xi_{0}$ is pure and the $\mathcal{T}_{\mathrm{k}}$-actions are all equivalent.

In Ref. 7 a weaker, but more explicit, statement was proven in complex dimension of 1 . Lemma 9.2 of Ref. 7 gives an explicit expression of $Q_{k, 0}^{*} T_{k}(a) Q_{k, 0}$ if $a \in A$ is smooth as

$$
Q_{k, 0}^{*} T_{k}(a) Q_{k, 0}=T_{0}\left(\mathcal{D}_{k}(a)\right),
$$

where $\mathcal{D}_{k}:=\mathrm{id}+\sum_{j=1}^{k} d_{j, k} \Delta^{j}$ for some explicit constants $d_{j, k}$ and $\Delta$ is the Laplacian on C.

For $i=1, \ldots, n$ we let $z_{i}: S^{2 n-1} \rightarrow \mathrm{C}$ denote the coordinate functions of the embedding $S^{2 n-1} \subseteq \mathrm{C}^{n}$. Clearly $z_{i} \in C\left(S^{2 n-1}\right)$.

Corollary 4.2: The operators $P_{\mathrm{k}} \lambda\left(z_{i}\right) P_{\mathrm{k}}^{*}$ together with $\mathcal{K}$ generate $\mathcal{T}_{\mathrm{k}}$ as a $C^{*}$-algebra.

Proof: Let $U$ denote the $C^{*}$-algebra generated by $P_{\mathrm{k}} \lambda\left(z_{i}\right) P_{\mathrm{k}}$ and $\mathcal{K}$. The $C^{*}$-algebra $\mathcal{T}_{\mathrm{k}}$ is constructed as the $C^{*}$-algebra generated by the linear space $P_{\mathrm{k}} \lambda\left(C\left(S^{2 n-1}\right)\right) P_{\mathrm{k}}$ because $P_{\mathrm{k}} \lambda(a) P_{\mathrm{k}}$ $-\widetilde{P}_{\mathrm{k}} \lambda(a) \widetilde{P}_{\mathrm{k}}^{*} \in \mathcal{K}$. So it is sufficient to prove $P_{\mathrm{k}} \lambda\left(C\left(S^{2 n-1}\right)\right) P_{\mathrm{k}} \subseteq U$. Given a function $a \in C\left(S^{2 n-1}\right)$ the Stone-Weierstrass theorem implies that there is a sequence of polynomials $R_{j}=R_{j}(z, \bar{z})$, such that $R_{j} \rightarrow a$ in $C\left(S^{2 n-1}\right)$. The functions $R_{j}$ are polynomials so it follows that

$$
P_{\mathrm{k}} \lambda\left(R_{j}\right) P_{\mathrm{k}}-R_{j}\left(P_{\mathrm{k}} \lambda(z) P_{\mathrm{k}}, P_{\mathrm{k}} \lambda\left(z^{*}\right) P_{\mathrm{k}}\right) \in \mathcal{K}
$$

and $\quad P_{\mathrm{k}} \lambda\left(R_{j}\right) P_{\mathrm{k}} \in U . \quad$ Finally, $\quad\left\|P_{\mathrm{k}} \lambda\left(R_{j}\right) P_{\mathrm{k}}-P_{\mathrm{k}} \lambda(a) P_{\mathrm{k}}\right\|_{\mathcal{B}\left(\mathcal{L}_{\mathrm{k}}\right)} \leq\left\|R_{j}-a\right\|_{C\left(S^{2 n-1}\right)} \quad$ which implies $P_{\mathrm{k}} \lambda(a) P_{\mathrm{k}} \in U$.

Corollary 4.3: The mapping $\beta_{\mathrm{k}}: C\left(S^{2 n-1}\right) \rightarrow \mathcal{C}\left(\mathcal{L}_{\mathrm{k}}\right)$ induced from $\widetilde{\beta}_{\mathrm{k}}$ is injective, so if $u \in A \otimes M_{N}$ the operator $T_{\mathrm{k}}(u)$ is Fredholm if and only if $\pi_{\partial}(u)$ is invertible.

Proof: Due to Eq. (6) in Theorem 4.1, the Corollary follows from Lemma 3.5. The proof of the second statement of the Corollary is proven in the same fashion as Proposition 3.6.

From the fact that the mapping $\beta_{\mathrm{k}}$ is injective it follows that the symbol mapping $\widetilde{P}_{\mathrm{k}} \lambda(a) \widetilde{P}_{\mathrm{k}}^{*} \mapsto a$ gives a well defined surjection $\sigma_{\mathrm{k}}: \mathcal{T}_{\mathrm{k}} \rightarrow C\left(S^{2 n-1}\right)$. Clearly the kernel of $\sigma_{\mathrm{k}}$ is nonzero and ker $\sigma_{\mathrm{k}} \subseteq \mathcal{K}$, so by Theorem $4.1 \mathrm{ker} \sigma_{\mathrm{k}}=\mathcal{K}$. Therefore, we can construct the exact sequence

$$
0 \rightarrow \mathcal{K} \rightarrow \mathcal{T}_{\mathrm{k}}{ }_{\mathrm{k}} \rightarrow C\left(S^{2 n-1}\right) \rightarrow 0 .
$$

A completely positive splitting of the symbol mapping $\sigma_{\mathrm{k}}: \mathcal{T}_{\mathrm{k}} \rightarrow C\left(S^{2 n-1}\right)$ is given by $a \mapsto \widetilde{P}_{\mathrm{k}} \lambda(a) \widetilde{P}_{\mathrm{k}}^{*}$.

The exact sequence (7) defines an extension class $\left[\mathcal{T}_{\mathrm{k}}\right] \in \operatorname{Ext}\left(C\left(S^{2 n-1}\right)\right)$. To read more about Ext, $K$-theory, and $K$-homology, we refer the reader to Appendix. Since $C\left(S^{2 n-1}\right)$ is a nuclear $C^{*}$-algebra there is an isomorphism $\operatorname{Ext}\left(C\left(S^{2 n-1}\right)\right) \cong K^{1}\left(C\left(S^{2 n-1}\right)\right)$ and we can describe the $K$-homology class of $\left[\mathcal{T}_{\mathrm{k}}\right]$ explicitly by a Fredholm module as follows; we let $\lambda: C\left(S^{2 n-1}\right)$ $\rightarrow \mathcal{B}\left(L^{2}\left(C^{n}\right)\right)$ be as in Eq. (4) and define the operator

$$
F_{\mathrm{k}}=\frac{\left(1+\widetilde{P}_{\mathrm{k}}\right)}{2},
$$

where $\widetilde{P}_{\mathrm{k}}$ is as in Eq. (5). Clearly, $\left(L^{2}\left(\mathrm{C}^{n}\right), \lambda, F_{\mathrm{k}}\right)$ defines a Fredholm module which represents the image of $\left[\mathcal{T}_{\mathrm{k}}\right]$ in $K^{1}\left(C\left(S^{2 n-1}\right)\right)$.

Corollary 4.4: The class $\left[\mathcal{T}_{\mathrm{k}}\right] \in \operatorname{Ext}\left(C\left(S^{2 n-1}\right)\right)$ is independent of $\mathrm{k}$.

Proof: The extension $\mathcal{T}_{\mathrm{k}}$ is equivalent to $\mathcal{T}_{\mathrm{k}^{\prime}}$ since it follows from Eq. (6) that the following diagram with exact rows commute 


$$
\begin{aligned}
0 \rightarrow & \mathcal{K} \rightarrow \mathcal{T}_{\mathrm{k}^{\prime}} \rightarrow C\left(S^{2 n-1}\right) \rightarrow 0 \\
& \downarrow_{\mathrm{Ad}\left(Q_{\mathrm{k}, \mathrm{k}^{\prime}}\right)} \downarrow_{\mathrm{Ad}\left(Q_{\mathrm{k}, \mathrm{k}^{\prime}}\right)} \| \\
0 \rightarrow & \mathcal{K} \rightarrow \mathcal{T}_{\mathrm{k}} \rightarrow C\left(S^{2 n-1}\right) \rightarrow 0 .
\end{aligned}
$$

So we know that $\left[\mathcal{T}_{\mathrm{k}}\right]$ is independent of $\mathrm{k}$, this implies that the index of $T_{\mathrm{k}}(u)$ for $u \in A \otimes M_{n}$ is independent of $\mathrm{k}$. But how do we calculate it? The index theorem that allows the calculation involves studying how the coordinate functions on $S^{2 n-1}$ act on the monomial base of $\mathcal{L}_{0}$. We will first review some theory of Toeplitz operators on the Bergman space and then study what happens in complex dimensions of 1 and 2.

The Bergman space on the unit ball $B_{n} \subseteq \mathrm{C}^{n}$ is defined as $A^{2}\left(B_{n}\right):=L^{2}\left(B_{n}\right) \cap \mathcal{O}\left(B_{n}\right)$, that is, holomorphic functions on $B_{n}$ which are square integrable. The Bergman space is a closed subspace of $L^{2}\left(B_{n}\right)$ and we will denote the orthogonal projection $L^{2}\left(B_{n}\right) \rightarrow A^{2}\left(B_{n}\right)$ by $P_{B}$.

The Bergman projection defines a $K$-homology class $\left[P_{B}\right] \in K^{1}\left(C\left(S^{2 n-1}\right)\right)$ in the same fashion as for the Landau projections. That is, for $a \in C\left(\bar{B}_{n}\right)$ the operator $\left[P_{B}, a\right] \in \mathcal{B}\left(L^{2}\left(B_{n}\right)\right)$ is compact. The reason that we can use $P_{B}$ to define a $K$-homology class for $S^{2 n-1}$ instead of $\overline{B_{n}}$ is analogously to above that $\left.P_{B} a\right|_{A^{2}\left(B_{n}\right)}$ is compact if and only if $a \in C_{0}\left(B_{n}\right)$, see more in Ref. 18. Thus, $\left.P_{B} a\right|_{A^{2}\left(B_{n}\right)}$ is Fredholm if and only if $\left.a\right|_{S^{2 n-1}}$ is invertible.

Furthermore, $\left[P_{B}, a\right]$ is compact. So $\left[P_{B}\right]$ is a well defined $K$-homology class in $K^{1}\left(C\left(S^{2 n-1}\right)\right)$. By Ref. 5 the following index formula holds for the Toeplitz operator $\left.P_{B} a\right|_{A^{2}\left(B_{n}\right)}$ if the symbol $a_{\partial}:=\left.a\right|_{S^{2 n-1}}$ is smooth:

$$
\operatorname{ind}\left(\left.P_{B} a\right|_{A^{2}\left(B_{n}\right.}\right)=\frac{-(n-1) !}{(2 n-1) !(2 \pi i)^{n}} \int_{S^{2 n-1}} \operatorname{tr}\left(\left(a_{\partial}^{-1} \mathrm{~d} a_{\partial}\right)^{2 n-1}\right) .
$$

This formula was also proven in Ref. 10 by an elegant use of Atiyah-Singers index theorem.

We will by $\mathcal{T}^{n}$ denote the $C^{*}$-algebra generated by $P_{B} C\left(\overline{B_{n}}\right) P_{B}$ in $\mathcal{B}\left(A^{2}\left(B_{n}\right)\right)$. The $K$-homology class $\left[P_{B}\right] \in K^{1}\left(C\left(S^{2 n-1}\right)\right)$ can be represented by the extension class $\left[\mathcal{T}^{n}\right] \in \operatorname{Ext}\left(C\left(S^{2 n-1}\right)\right)$ defined by means of the short exact sequence,

$$
0 \rightarrow \mathcal{K} \rightarrow \mathcal{T}^{n} \stackrel{\sigma^{n}}{\rightarrow} C\left(S^{2 n-1}\right) \rightarrow 0
$$

\section{THE SPECIAL CASES $\mathrm{C}$ and $\mathrm{C}^{2}$}

In this section we will study the special cases of complex dimensions of 1 and 2. Dimension 1 has been studied previously in Ref. 2 and provides a simpler picture than in higher dimensions. In the one-dimensional case we have that $K_{1}(C(T)) \cong \mathbb{Z}$ and we can take the coordinate function $z: T \rightarrow C$ to be a generator. So when we want to determine the class $\left[\mathcal{T}_{k}\right]$ we only need to calculate the index of $P_{k} \lambda(z) P_{k}$, where $\lambda$ is as in Eq. (4). We recall the following proposition from Ref. 2.

Proposition 5.1: (Proposition 7.3 from Ref. 2) For any $k \in \mathbb{N}$ we have that

$$
\operatorname{ind}\left(P_{k} \lambda(z) P_{k}\right)=-1 \text {. }
$$

The method used in Ref. 2 to prove this Proposition was to show that in a suitable basis $P_{k} \lambda(z) P_{k}$ was up to some coefficients a unilateral shift. In higher dimension the proof is based on similar ideas.

Theorem 5.2: For $n=1$ there is an isomorphism $\mathcal{T}_{k} \cong \mathcal{T}^{1}$ making $\left[\mathcal{T}_{k}\right]=\left[\mathcal{T}^{1}\right] \in K^{1}(C(\mathbb{T}))$.

Proof: By Proposition 7.3 of Ref. 2,

$$
\left[\mathcal{T}_{k}\right] \cdot[u]=\operatorname{ind}\left(P_{k} \lambda(u) P_{k}\right)=-\operatorname{wind}(u)=\left[\mathcal{T}^{1}\right] .[u]
$$

for an invertible function $u \in C(\mathbb{T})$. Here wind $(u)$ denotes the winding number of $u$ which is defined for smooth $u$ as 


$$
\operatorname{wind}(u):=\frac{1}{2 \pi i} \int_{\mathbb{T}} u^{-1} \mathrm{~d} u
$$

and defines an isomorphism $K_{1}(C(T)) \rightarrow Z$ Z By the universal coefficient theorem for $K K$-theory (see Theorem 4.2 of Ref. 15) the mapping

$$
K^{1}(C(\mathbb{T})) \rightarrow \operatorname{Hom}\left(K_{1}(C(\mathbb{T})), \mathbb{Z}\right)
$$

is an isomorphism so Eq. (10) implies that $\left[\mathcal{T}_{k}\right]=\left[\mathcal{T}^{1}\right]$.

By Theorem 13 of Ref. 8, the short exact sequence $0 \rightarrow \mathcal{K} \rightarrow \mathcal{T}_{k} \rightarrow C(\mathbb{T}) \rightarrow 0$ is characterized by an isometry $v$, such that $v v^{*}-1$ is compact and $\mathcal{T}_{k}$ is generated by $v$. Then $z \mapsto v$ defines a splitting and the symbol mapping $\mathcal{T}_{k} \rightarrow C(T)$ is just $v \mapsto z$. By Eq. (10), $1-v v^{*}$ is a rank 1 projection, so the theorem follows.

Also in dimension of 2 we can find a generator for the odd $K$-theory. As generator for $K_{1}\left(C\left(S^{3}\right)\right) \cong \mathbb{Z}$ we can take the diffeomorphism $u: S^{3} \rightarrow \mathrm{SU}(2)$ defined as

$$
u\left(z_{1}, z_{2}\right):=\left(\begin{array}{cc}
z_{1} & z_{2} \\
-\bar{z}_{2} & \bar{z}_{1}
\end{array}\right) .
$$

Proposition 5.3: The extension class $\left[\mathcal{T}^{2}\right]$ generate $K^{1}\left(C\left(S^{3}\right)\right)$ and $[u]$ generate $K_{1}\left(C\left(S^{3}\right)\right)$.

Proof: Recalling that $P_{B}$ denotes the Bergman projection we will start by calculating the index of the Toeplitz operator $P_{B} u P_{B}: A^{2}\left(B_{2}\right) \otimes \mathrm{C}^{2} \rightarrow A^{2}\left(B_{2}\right) \otimes \mathrm{C}^{2}$. Using the index theorem by Boutet de Monvel $^{5}$ reviewed above in Eq. (8), the following index formula holds for smooth $u$ :

$$
\operatorname{ind}\left(P_{B} u P_{B}\right)=-\frac{1}{3 !(2 \pi i)^{2}} \int_{S^{3}} \operatorname{tr}\left(\left(u^{*} \mathrm{~d} u\right)^{3}\right) .
$$

A straightforward calculation gives that

$$
\operatorname{tr}\left(\left(u^{*} \mathrm{~d} u\right)^{3}\right)=3\left(z_{1} \mathrm{~d} \bar{z}_{1}-\bar{z}_{1} \mathrm{~d} z_{1}\right) \wedge \mathrm{d} z_{2} \wedge \mathrm{d} \bar{z}_{2}+3\left(z_{2} \mathrm{~d} \bar{z}_{2}-\bar{z}_{2} \mathrm{~d} z_{2}\right) \wedge \mathrm{d} z_{1} \wedge \mathrm{d} \bar{z}_{1} .
$$

Invoking Stokes theorem on Eq. (11) gives that

$$
\begin{aligned}
-\frac{1}{3 !(2 \pi i)^{2}} \int_{S^{3}} \operatorname{tr}\left(\left(u^{*} \mathrm{~d} u\right)^{3}\right) & =\frac{1}{48 \cdot \operatorname{vol}\left(B_{2}\right)} \int_{B_{2}} \mathrm{~d} \operatorname{tr}\left(\left(u^{*} \mathrm{~d} u\right)^{3}\right) \\
& =\frac{1}{4 \cdot \operatorname{vol}\left(B_{2}\right)} \int_{B_{2}} \mathrm{~d} z_{1} \wedge \mathrm{d} \bar{z}_{1} \wedge \mathrm{d} z_{2} \wedge \mathrm{d} \bar{z}_{2} \\
& =-\frac{1}{\operatorname{vol}\left(B_{2}\right)} \int_{B_{2}} \mathrm{~d} V=-1 .
\end{aligned}
$$

This equation shows that

$$
\left[\mathcal{T}^{2}\right] .[u]=\operatorname{ind}\left(P_{B} u P_{B}\right)=-1 .
$$

Consider the split-exact sequence $0 \rightarrow C_{0}\left(\mathbb{R}^{3}\right) \rightarrow C\left(S^{3}\right) \rightarrow \mathrm{C} \rightarrow 0$ where the mapping $C\left(S^{3}\right) \rightarrow \mathrm{C}$ is point evaluation. Since the sequence splits, and $K_{1}(\mathrm{C})=K^{1}(\mathrm{C})=0$ the embedding $C_{0}\left(\mathbb{R}^{3}\right) \rightarrow C\left(S^{3}\right)$ induces isomorphisms $K_{1}\left(C\left(S^{3}\right)\right) \cong K_{1}\left(C_{0}\left(\mathbb{R}^{3}\right)\right)=\mathbb{Z}$ and $K^{1}\left(C\left(S^{3}\right)\right) \cong K^{1}\left(C_{0}\left(\mathbb{R}^{3}\right)\right)$ $=\mathbb{Z}$. So the Kasparov product $K_{1}\left(C\left(S^{3}\right)\right) \times K^{1}\left(C\left(S^{3}\right)\right) \rightarrow \mathbb{Z}$ is just a pairing $\mathbb{Z} \times \mathbb{Z} \rightarrow \mathbb{Z}$, and since $\left[\mathcal{T}^{2}\right]$. $[u]=-1$ it follows that $\left[\mathcal{T}^{2}\right]$ generates $K^{1}\left(C\left(S^{3}\right)\right)$ and $[u]$ generates $K_{1}\left(C\left(S^{3}\right)\right)$.

Theorem 5.4: For any $\mathrm{k} \in \mathbb{N}^{2}$ we have

$$
\operatorname{ind}\left(P_{\mathrm{k}} \lambda(u) P_{\mathrm{k}}\right)=-1 .
$$

Therefore, $\left[\mathcal{T}^{2}\right]=\left[\mathcal{T}_{\mathrm{k}}\right]$.

Proof: If Eq. (13) holds, $\left[\mathcal{T}^{2}\right]=\left[\mathcal{T}_{\mathrm{k}}\right]$ follows directly from Eq. (12) using the universal coef- 
ficient theorem for $K K$-theory (see Theorem 4.2 of Ref. 15). This is a consequence of the fact that the natural mapping,

$$
K^{1}\left(C\left(S^{3}\right)\right) \rightarrow \operatorname{Hom}\left(K_{1}\left(C\left(S^{3}\right)\right), Z\right),
$$

is an isomorphism. The injectivity of this map implies that if $\left[\mathcal{T}^{2}\right] \cdot[u]=\left[\mathcal{T}_{\mathrm{k}}\right] \cdot[u]$ for a generator $[u]$ then $\left[\mathcal{T}^{2}\right]=\left[\mathcal{T}_{\mathrm{k}}\right]$.

To prove Eq. (13) we take $\mathrm{k}=0$, since Corollary 4.4 implies that the integer $\operatorname{ind}\left(P_{\mathrm{k}} \lambda(u) P_{\mathrm{k}}\right)$ is independent of $\mathrm{k}$. We claim that $P_{0} \lambda(u) P_{0}$ is an injective operator and the cokernel of $P_{0} \lambda(u) P_{0}$ is spanned by the $\mathrm{C}^{2}$-valued function $z \mapsto \mathrm{e}^{-|z|^{2} / 4} \oplus 0$. This statement will prove the theorem.

To prove that $P_{0} \lambda(u) P_{0}$ is injective, assume $f \in \operatorname{ker}\left(P_{0} \lambda(u) P_{0}\right)$. Define the functions

$$
\xi^{\mathrm{m}}(z):=z^{\mathrm{m}} \mathrm{e}^{-|z|^{2} / 4}
$$

for $m \in \mathbb{N}^{2}$. The functions $\xi^{\mathrm{m}}$ form an orthogonal basis for $\mathcal{L}_{0}$ by Theorem 1.63 of Ref. 9. Expand the function $f$ in an $L^{2}$-convergent series,

$$
f=\sum_{\mathrm{m} \in \mathbb{N}^{2}} c_{\mathrm{m}} \xi^{\mathrm{m}},
$$

where $c_{\mathrm{m}}=c_{\mathrm{m}}^{(1)} \oplus c_{\mathrm{m}}^{(2)} \in \mathrm{C}^{2}$. Since $f \in \operatorname{ker}\left(P_{0} \lambda(u) P_{0}\right)$ we have the following orthogonality condition:

$$
\begin{aligned}
0 & =\left\langle\xi^{\mathrm{m}^{\prime}} \oplus 0, \lambda(u) f\right\rangle \\
& =\sum_{\mathrm{m}} \int_{\mathrm{C}^{2}}\left(c_{\mathrm{m}}^{(1)} \frac{\bar{z}^{\mathrm{m}^{\prime}} z^{\mathrm{m}+e_{1}}}{|z|}+c_{\mathrm{m}}^{(2)} \frac{\bar{z}^{\mathrm{m}^{\prime}} z^{\mathrm{m}+e_{2}}}{|z|}\right) \mathrm{e}^{|z|^{2} / 2} \mathrm{~d} V \\
& =\sum_{\mathrm{m}} t_{\mathrm{m}, \mathrm{m}^{\prime}} \int_{S^{3}}\left(c_{\mathrm{m}}^{(1)} \bar{z}^{\mathrm{m}^{\prime}} z^{\mathrm{m}+e_{1}}+c_{\mathrm{m}}^{(2)} \bar{z}^{\mathrm{m}^{\prime}} z^{\mathrm{m}+e_{2}}\right) \mathrm{d} S,
\end{aligned}
$$

for some coefficients $t_{\mathrm{m}, \mathrm{m}^{\prime}}$, for a detailed calculation of $t_{\mathrm{m}, \mathrm{m}^{\prime}}$ see below in Proposition 6.1. Using that the functions $\xi^{\mathrm{m}}$ are orthogonal we obtain that there exist a $C_{\mathrm{m}}>0$, such that

$$
c_{\mathrm{m}-e_{1}}^{(1)}=-C_{\mathrm{m}} c_{\mathrm{m}-e_{2}}^{(2)} .
$$

On the other hand, we have

$$
\begin{aligned}
0 & =\left\langle 0 \oplus \xi^{\mathrm{m}^{\prime}}, \lambda(u) f\right\rangle \\
& =\sum_{\mathrm{m}} \int_{\mathbb{C}^{2}}\left(-c_{\mathrm{m}}^{(1)} \frac{z^{\mathrm{m}^{\prime}+e_{2}} z^{\mathrm{m}}}{|z|}+c_{\mathrm{m}}^{(2)} \frac{z^{\mathrm{m}^{\prime}+e_{1}} z^{\mathrm{m}}}{|z|}\right) \mathrm{e}^{|z|^{2} / 2} \mathrm{~d} V \\
& =\sum_{\mathrm{m}} t_{\mathrm{m}, \mathrm{m}^{\prime}} \int_{S^{3}}\left(-c_{\mathrm{m}}^{(1)} z^{\mathrm{m}^{\prime}+e_{2}} z^{\mathrm{m}}+c_{\mathrm{m}}^{(2)} \bar{z}^{\mathrm{m}^{\prime}+e_{1}} z^{\mathrm{m}}\right) \mathrm{d} S .
\end{aligned}
$$

Again using orthogonality of the functions $\xi^{\mathrm{m}}$ we obtain that there is a $C_{\mathrm{m}}^{\prime}>0$, such that

$$
c_{\mathrm{m}+e_{2}}^{(1)}=C_{\mathrm{m}}^{\prime} c_{\mathrm{m}+e_{1}}^{(2)} .
$$

Equation (14) implies $c_{\mathrm{m}}^{(1)}=0$ for $m_{2}=0$. For $m_{2}>0$ Eq. (14) implies

$$
c_{\mathrm{m}}^{(1)}=-C_{\mathrm{m}+e_{1}} c_{\mathrm{m}-e_{2}+e_{1}}^{(2)} .
$$

Then Eq. (15) for $\mathrm{m}-e_{2}$ gives 


$$
c_{\mathrm{m}}^{(1)}\left(1+\frac{C_{\mathrm{m}+e_{1}}}{C_{\mathrm{m}-e_{2}}^{\prime}}\right)=0 .
$$

So $c_{\mathrm{m}}^{(1)}=0$ for all $\mathrm{m}$. Equation (14) implies $c_{\mathrm{m}}^{(2)}=0$ for all $\mathrm{m}$. Thus, $f=0$ and $\operatorname{ker}\left(P_{0} \lambda(u) P_{0}\right)=0$.

The second statement, that the cokernel of $P_{0} \lambda(u) P_{0}$ is spanned by the $\mathrm{C}^{2}$-valued function

$$
z \mapsto \mathrm{e}^{-|z|^{2} / 4} \oplus 0
$$

is proven analogously. There is a natural isomorphism,

$$
\text { coker } P_{0} \lambda(u) P_{0} \cong\left(\operatorname{im} P_{0} \lambda(u) P_{0}\right)^{\perp}=\operatorname{ker} P_{0} \lambda\left(u^{*}\right) P_{0} .
$$

Analogously to the reasoning above, for $g \in \operatorname{ker} P_{0} \lambda\left(u^{*}\right) P_{0}$ we expand the function $g$ in an $L^{2}$-convergent series,

$$
g=\sum_{\mathrm{m} \in \mathbb{N}^{2}} d_{\mathrm{m}} \xi^{\mathrm{m}}
$$

where $d_{\mathrm{m}}=d_{\mathrm{m}}^{(1)} \oplus d_{\mathrm{m}}^{(2)} \in \mathbb{C}^{2}$. After taking scalar product by $\xi_{\mathrm{m}^{\prime}}$, for some $D_{\mathrm{m}}, D_{\mathrm{m}}^{\prime}>0$ we obtain the following conditions on the coefficients:

$$
d_{\mathrm{m}+e_{1}}^{(1)}=D_{\mathrm{m}} d_{\mathrm{m}-e_{2}}^{(2)}
$$

and

$$
d_{\mathrm{m}+e_{2}}^{(1)}=-D_{\mathrm{m}}^{\prime} d_{\mathrm{m}-e_{1}}^{(2)} .
$$

The second of these equations implies $d_{\mathrm{m}}^{(1)}=0$ for $m_{1}=0$ and $m_{2}>0$. Also, the first of these equations implies $d_{\mathrm{m}}^{(1)}=0$ for $m_{2}=0$ and $m_{1}>0$. For $m_{1}, m_{2}>0$, putting in $m-e_{1}$ in the first equation, gives

$$
d_{\mathrm{m}}^{(1)}=D_{\mathrm{m}-e_{1}} d_{\mathrm{m}-e_{1}-e_{2}}^{(2)} .
$$

Finally, combining this relation with the second equation for $m-e_{2}$, we obtain

$$
d_{\mathrm{m}}^{(1)}\left(1+\frac{D_{\mathrm{m}-e_{1}}}{D_{\mathrm{m}-e_{2}}^{\prime}}\right)=0 \text { for } m_{1}, m_{2}>0 .
$$

Therefore, $d_{\mathrm{m}}^{(1)}=0$ for all $\mathrm{m} \neq 0$. The equations in (16) imply $d_{\mathrm{m}}^{(2)}=0$ for all $\mathrm{m}$. However, the function $z \mapsto \mathrm{e}^{-|z|^{2} / 4} \oplus 0$, corresponding to $d_{0}^{(1)}=1$, is in the $\operatorname{space} \operatorname{ker}\left(P_{0} \lambda\left(u^{*}\right) P_{0}\right)$ which completes the proof.

\section{THE INDEX FORMULA ON THE PARTICULAR LANDAU LEVELS}

In this section we will prove an index formula for the particular Landau levels. On $S^{2 n-1}$ we have the complex coordinates $z_{1}, \ldots, z_{n}$ and we denote by $Z_{1}, \ldots, Z_{n}$ the image of these coordinate functions under the representation $\lambda$ which was defined in Eq. (4). So $Z_{i}$ is the operator on $L^{2}\left(C^{n}\right)$ given by multiplication by the almost everywhere defined function $z \mapsto z_{i} /|z|$. Consider the polar decompositions,

$$
P_{0} Z_{i} P_{0}=V_{i, 0} S_{i, 0},
$$

where $V_{i, 0}$ are partial isometries and $S_{i, 0}>0$. An orthonormal basis for $\mathcal{L}_{0}$ is given by 


$$
\eta_{\mathrm{m}}(z):=\frac{z^{\mathrm{m}} \mathrm{e}^{-|z|^{2 / 4}}}{\sqrt{\pi^{n} 2^{|\mathrm{m}|+n} \mathrm{~m} !}},
$$

see more in Ref. 9.

Proposition 6.1: The operator $V_{i, 0}$ is an isometry described by the equation

$$
V_{i, 0} \eta_{\mathrm{m}}=\eta_{\mathrm{m}+e_{i}}
$$

and the operator $S_{i, 0}$ is diagonal in the basis $\eta_{\mathrm{m}}$ with eigenvalues given by

$$
\lambda_{i, \mathrm{~m}}^{\eta}=\Gamma\left(|\mathrm{m}|+n+\frac{1}{2}\right) \frac{\sqrt{m_{i}+1}}{(|\mathrm{~m}|+n) !} .
$$

Proof: For $\mathrm{m}, \mathrm{m}^{\prime} \in \mathbb{N}$ we have

$$
\begin{aligned}
\left\langle\eta_{\mathrm{m}^{\prime}}, Z_{i} \eta_{\mathrm{m}}\right\rangle & =\int_{\mathrm{C}^{n}} \frac{1}{\pi^{n} \sqrt{2^{\left|\mathrm{m}+\mathrm{m}^{\prime}\right|+2 n \mathrm{~m} ! \mathrm{m}^{\prime}} !}} \frac{\bar{z}^{\mathrm{m}^{\prime}} z^{\mathrm{m}+e_{i}}}{|z|} \mathrm{e}^{-|z|^{2} / 2} \mathrm{~d} V \\
& =\frac{1}{\pi^{n} \sqrt{2^{\left|\mathrm{m}+\mathrm{m}^{\prime}\right|+2 n} \mathrm{~m} ! \mathrm{m}^{\prime} !}} \int_{0}^{\infty} r^{|\mathrm{m}|+\left|\mathrm{m}^{\prime}\right|+n-1} \mathrm{e}^{-r^{2} / 2} \mathrm{~d} r \int_{S^{2 n-1}} \bar{z}^{\mathrm{m}^{\prime}} z^{\mathrm{m}+e_{i}} \mathrm{~d} S \\
& =\delta_{\mathrm{m}^{\prime}, \mathrm{m}+e_{i}} \frac{\Gamma\left(|\mathrm{m}|+n+\frac{1}{2}\right)}{2 \pi^{n} \mathrm{~m} ! \sqrt{\left(\mathrm{m}_{j}+1\right)}} \int_{S^{2 n-1}} \bar{z}^{\mathrm{m}^{\prime}} z^{\mathrm{m}+e_{i}} \mathrm{~d} S \\
& =\delta_{\mathrm{m}^{\prime}, \mathrm{m}+e_{i}} \Gamma\left(|\mathrm{m}|+n+\frac{1}{2}\right) \frac{\sqrt{m_{i}+1}}{(|\mathrm{~m}|+n) !}
\end{aligned}
$$

It follows that $V_{i, 0} \eta_{\mathrm{m}}=\eta_{\mathrm{m}+e_{i}}$ and $S_{i, 0} \eta_{\mathrm{m}}=\lambda_{i, \mathrm{~m}}^{\eta} \eta_{\mathrm{m}}$, where $\lambda_{i, \mathrm{~m}}^{\eta}$ is as in Eq. (18).

On the other hand, we can, just as on $\mathcal{L}_{0}$, let $\widetilde{Z}_{1}, \ldots, \widetilde{Z}_{n} \in \mathcal{B}\left(L^{2}\left(B_{n}\right)\right)$ be the operators on $L^{2}\left(B_{n}\right)$ defined by the multiplication by the almost everywhere defined function $z \mapsto z_{i} /|z|$. Consider the polar decompositions,

$$
P_{B} \widetilde{Z}_{i} P_{B}=V_{i, B} S_{i, B}
$$

where again $V_{i, B}$ are partial isometries and $S_{i, B}>0$. An orthonormal basis for $A^{2}\left(B_{n}\right)$ is given by

$$
\mu_{\mathrm{m}}(z):=\pi^{-n / 2} \sqrt{\frac{(n+|\mathrm{m}|) !}{\mathrm{m} !}} z^{\mathrm{m}} .
$$

Similar to the lowest Landau level, the partial isometries $V_{i, B}$ are just shifts in this basis:

Proposition 6.2: The operator $V_{i, B}$ is an isometry described by the equation

$$
V_{i, B} \mu_{\mathrm{m}}=\mu_{\mathrm{m}+e_{i}}
$$

and the operator $S_{i, B}$ is diagonal in the basis $\mu_{\mathrm{m}}$ with eigenvalues given by

$$
\lambda_{i, \mathrm{~m}}^{\mu}=\frac{\sqrt{m_{i}+1}}{\sqrt{n+|\mathrm{m}|+1}} .
$$

Proof: The proof is the analogous to that of Proposition 6.1. For $m, m^{\prime} \in \mathbb{N}$ we have 


$$
\begin{aligned}
\left\langle\mu_{\mathrm{m}^{\prime}}, \tilde{Z}_{i} \mu_{\mathrm{m}}\right\rangle & =\int_{B_{n}} \pi^{-n} \sqrt{\frac{(n+|\mathrm{m}|) !\left(n+\left|\mathrm{m}^{\prime}\right|\right) !}{\mathrm{m} ! \mathrm{m}^{\prime} !}} \frac{z^{\mathrm{m}^{\prime}} z^{\mathrm{m}+e_{i}}}{|z|} \mathrm{d} V \\
& =\pi^{-n} \sqrt{\frac{(n+|\mathrm{m}|) !\left(n+\left|\mathrm{m}^{\prime}\right|\right) !}{\mathrm{m} ! \mathrm{m}^{\prime} !}} \int_{0}^{1} r^{\left|\mathrm{m}^{\mathrm{m}}\right|+\mathrm{m}^{\prime} \mid+2 n-1} \mathrm{~d} r \int_{S^{2 n-1}} z^{\mathrm{m}^{\prime}} z^{\mathrm{m}+e_{i}} \mathrm{~d} S \\
& =\delta_{\mathrm{m}^{\prime}, \mathrm{m}+e_{i}} \frac{(n+|\mathrm{m}|) ! \sqrt{n+|\mathrm{m}|+1}}{(2|\mathrm{~m}|+2 n) \mathrm{m} ! \sqrt{m_{i}+1}} \int_{S^{2 n-1}} z^{\mathrm{m}^{\prime}} z^{\mathrm{m}+e_{i}} \mathrm{~d} S \\
& =\delta_{\mathrm{m}^{\prime}, \mathrm{m}+e_{i}} \frac{\sqrt{m_{i}+1}}{\sqrt{n+|\mathrm{m}|+1}} .
\end{aligned}
$$

It follows that $V_{i, B} \mu_{\mathrm{m}}=\mu_{\mathrm{m}+e_{i}}$ and $S_{i, B} \mu_{\mathrm{m}}=\lambda_{i, \mathrm{~m}}^{\mu} \mu_{\mathrm{m}}$, where the eigenvalues $\lambda_{i, \mathrm{~m}}^{\mu}$ are given in Eq. (19).

Lemma 6.3: If a is a real number then

$$
\frac{\Gamma(x+a)}{\Gamma(x)}=x^{a}+O\left(x^{-1+a}\right) \quad \text { as } \quad x \rightarrow+\infty .
$$

Proof: By Stirling's formula

$$
\ln \Gamma(x)=\left(x-\frac{1}{2}\right) \ln x-x+\frac{\ln 2 \pi}{2}+O\left(x^{-1}\right) .
$$

After Taylor expanding $\ln \Gamma(x+a)$ around $a=0$ we obtain that

$$
\ln \Gamma(x+a)-\ln \Gamma(x)=a \ln x+O\left(x^{-1}\right) .
$$

Lemma 6.4: With the unitary $U: A^{2}\left(B_{n}\right) \rightarrow \mathcal{L}_{0}$ defined by $\mu_{\mathrm{m}} \mapsto \eta_{\mathrm{m}}$, the operators $S_{i, 0}$ and $S_{i, B}$ satisfy

$$
U^{*} S_{i, 0} U-S_{i, B} \in \mathcal{K} .
$$

Proof: The operators $U^{*} S_{i, 0} U$ and $S_{i, B}$ are both diagonal in the basis $\mu_{\mathrm{m}}$. So it is sufficient to prove that $\left|\lambda_{\mathrm{m}}^{\eta}-\lambda_{\mathrm{m}}^{\mu}\right| \rightarrow 0$. The proof of this statement is based on the estimate from Lemma 6.3. When $|\mathrm{m}| \rightarrow \infty$, Lemma 6.3 implies

$$
\begin{aligned}
\left|\lambda_{\mathrm{m}}^{\eta}-\lambda_{\mathrm{m}}^{\mu}\right| & =\left|\frac{\Gamma\left(|\mathrm{m}|+n+\frac{1}{2}\right) \sqrt{m_{i}+1}}{(|\mathrm{~m}|+n) !}-\frac{\sqrt{m_{i}+1}}{\sqrt{|\mathrm{m}|+n-1}}\right| \\
& =\sqrt{m_{i}+1}\left|\frac{\Gamma\left((|\mathrm{m}|+n+1)-\frac{1}{2}\right)}{\Gamma(|\mathrm{m}|+n+1)}-(|\mathrm{m}|+n-1)^{-1 / 2}\right|=O\left(|\mathrm{~m}|^{-1}\right) .
\end{aligned}
$$

Therefore, we have that $U^{*} S_{i, 0} U-S_{i, B} \in \mathcal{L}^{n+}\left(A^{2}\left(B_{n}\right)\right)$, the $n$th Dixmier ideal. In particular, $U^{*} S_{i, 0} U-S_{i, B}$ is compact.

Theorem 6.5: The unitary $U$ induces an isomorphism $\operatorname{Ad}(U): \mathcal{T}_{0} \rightarrow \mathcal{T}^{n}$, such that

$$
\sigma^{n} \circ \operatorname{Ad}(U)=\sigma_{0},
$$

where $\sigma^{n}$ and $\sigma_{0}$ are the symbol mappings.

Proof: Lemma 6.4 and the Propositions 6.1 and 6.2 imply 


$$
U^{*}\left(P_{0} Z_{i} P_{0}\right) U=P_{B} \widetilde{Z}_{i} P_{B}+K_{i}
$$

for some compact operators $K_{i}$. Since $\mathcal{T}^{n}$ contains the compact operators, $U^{*}\left(P_{0} Z_{i} P_{0}\right) U \in \mathcal{T}^{n}$. Corollary 4.2 therefore implies $U^{*} \mathcal{T}_{0} U \subseteq \mathcal{T}^{n}$. Theorem 4.1 states that $\mathcal{T}_{0}$ acts irreducibly on $\mathcal{L}_{0}$, so $U^{*} \mathcal{T}_{0} U$ acts irreducibly on $A^{2}\left(B_{n}\right)$. Therefore, $\mathcal{K} \subseteq U^{*} \mathcal{T}_{0} U$ and $P_{B} \widetilde{Z}_{i} P_{B} \in U^{*} \mathcal{T}_{0} U$. The operators $P_{B} \widetilde{Z}_{i} P_{B}$ together with $\mathcal{K}$ generate $\mathcal{T}^{n}$ so $U^{*} \mathcal{T}_{0} U \supseteq \mathcal{T}^{n}$. The relation $\sigma^{n} \circ \operatorname{Ad}(U)=\sigma_{0}$ holds since by Eq. (20) it holds on the generators of $C\left(S^{2 n-1}\right)$.

Corollary 6.6: Let $\left[\mathcal{T}^{n}\right] \in \operatorname{Ext}\left(C\left(S^{2 n-1}\right)\right)$ denote the Toeplitz quantization of the Bergman space defined in Eq. (9) and $\left[\mathcal{T}_{\mathrm{k}}\right] \in \operatorname{Ext}\left(C\left(S^{2 n-1}\right)\right)$ the Toeplitz quantization of the particular Landau level of height $\mathrm{k}$ defined in Eq. (7). Then

$$
\left[\mathcal{T}^{n}\right]=\left[\mathcal{T}_{\mathrm{k}}\right] .
$$

So for $u \in A \otimes M_{N}$, such that $u_{\partial}:=\pi_{\partial}(u)$ is invertible and smooth

$$
\operatorname{ind}\left(\left.P_{\mathrm{k}} u\right|_{\mathcal{L}_{\mathrm{k}} \otimes \mathrm{C}^{N}}\right)=\frac{-(n-1) !}{(2 n-1) !(2 \pi i)^{n}} \int_{S^{2 n-1}} \operatorname{tr}\left(\left(u_{\partial}^{-1} \mathrm{~d} u_{\partial}\right)^{2 n-1}\right) .
$$

Proof: By Corollary 4.4 the class $\left[\mathcal{T}_{\mathrm{k}}\right]$ is independent of $\mathrm{k}$, so take $\mathrm{k}=0$. In this case Theorem 6.5 implies that the unitary $U$ makes the following diagram commutative:

$$
\begin{aligned}
0 & \rightarrow \mathcal{K} \rightarrow \mathcal{T}_{0} \stackrel{\sigma_{0}}{\rightarrow} C\left(S^{2 n-1}\right) \rightarrow 0 \\
& \downarrow \operatorname{Ad}(U) \downarrow \operatorname{Ad}(U) \quad \| \\
0 \rightarrow & \mathcal{K} \rightarrow \mathcal{T}^{n} \stackrel{\sigma_{n}}{\rightarrow} C\left(S^{2 n-1}\right) \rightarrow 0 .
\end{aligned}
$$

Therefore, $\left[\mathcal{T}^{n}\right]=\left[\mathcal{T}_{0}\right]=\left[\mathcal{T}_{\mathrm{k}}\right]$ and the index formula (21) follows from Ref. 10.

\section{APPENDIX: INDEX AND $K$-theory}

$K$-theory was first introduced by Grothendieck in his formulation of the Riemann-Roch theorem in 1957. In 1964 Atiyah and Hirzebruch introduced topological $K$-theory, see Ref. 1. Atiyah later used $K$-theory in the proof of the Atiyah-Singer index theorem. A more general framework to work with index problems was introduced by Kasparov in 1980 via his $K K$-theory, to read more about $K K$-theory, see Ref. 11. We will give a short introduction to odd $K$-theory and odd $K$-homology of $C^{*}$-algebras. Finally, we will show how odd $K$-theory and odd $K$-homology combine into index theory. The main references of this appendix is Ref. 4 for the odd $K$-theory and Ref. 11 for the odd $K$-homology.

The odd $K$-theory is a homological tool for dealing with symbols of Toeplitz operators in a $C^{*}$-algebra. There are several equivalent descriptions of odd $K$-theory, see more in Chap. 8 of Ref. 4. The description in this appendix is chosen to fit with index theory. We will by $A$ denote a separable $C^{*}$-algebra and use the notation $\widetilde{A}$ for the unitalization of $A$. We refer the reader unfamiliar with $C^{*}$-algebras to Ref. 13 . The reluctant reader who do not wish to become familiar with $C^{*}$-algebras could replace any occurrence of $A$ by $C_{0}(X)$, the continuous functions on a locally compact Hausdorff space $X$ vanishing at infinity. In the setting where $A=C_{0}(X)$ the unitalization can be described as $\widetilde{A}=C(\widetilde{X})$, where $\widetilde{X}$ is the one-point compactification of $X$.

The group $\mathrm{GL}_{N}(A)$ is defined as the invertible matrices in $\tilde{A} \otimes M_{N}(\mathrm{C})$ and $\mathrm{GL}_{\infty}(A):=\lim _{\rightarrow} \mathrm{GL}_{N}(A)$, where we embed $\mathrm{GL}_{N}(A) \rightarrow \mathrm{GL}_{N+1}(A)$ by $x \mapsto x \oplus 1$. The group $\mathrm{GL}_{\infty}(A)$ 
becomes a topological group in the inductive limit topology. We will denote the identity component of $\mathrm{GL}_{\infty}(A)$ by $\mathrm{GL}_{\infty}(A)_{0}$ which by standard theory is a normal subgroup. The odd $K$-theory is defined as in Definition 8.1.1 of Ref. 4 as

$$
K_{1}(A):=\mathrm{GL}_{\infty}(A) / \mathrm{GL}_{\infty}(A)_{0} .
$$

So the invariant $K_{1}(A)$ is the group of equivalence classes of invertible matrices over $\tilde{A}$, the equivalence relation involves stable homotopy. By Proposition 8.1.3 of Ref. 4 the group $K_{1}(A)$ is Abelian so the odd $K$-theory can be viewed as a covariant functor on the category of $C^{*}$-algebras to the category of Abelian groups. This functor has several useful properties making it possible to calculate it for many explicit $C^{*}$-algebras. The functor $K_{1}$ is homotopy invariant (Theorem 8.2.2 of Ref. 4) and half-exact (Corollary 8.2.3 of Ref. 4).

On the dual side of $K_{1}$ we have $K^{1}$, the odd $K$-homology. An element of $K^{1}(A)$ is an equivalence class of a quantization of $A$. By a quantization we mean a linear mapping from $A$ to the algebra of bounded operators on a Hilbert space multiplicative up to a compact operator as an error. A convenient description of $K^{1}$ is via analytic $K$-homology. First we need the notion of a bounded odd Fredholm module, taken from Chap. 2 of Ref. 11. A bounded odd Fredholm module over a $C^{*}$-algebra $A$ is a pair $(\pi, F)$, where $\pi: A \rightarrow \mathcal{B}(\mathcal{H})$ is a representation and $F \in \mathcal{B}(\mathcal{H})$ is an operator satisfying that

$$
\pi(a)\left(F^{2}-1\right), \pi(a)\left(F^{*}-F\right),[F, \pi(a)] \in \mathcal{K}(\mathcal{H}) \quad \text { for all } a \in A .
$$

Two bounded odd Fredholm modules $\left(\pi_{0}, F_{0}\right)$ and $\left(\pi_{1}, F_{1}\right)$ are said to be isomorphic if there exist a unitary $U \in \mathcal{B}(\mathcal{H})$, such that $\pi_{0}(a)=U^{*} \pi_{1}(a) U$ and $F_{0}=U^{*} F_{1} U$. We will denote the set of isomorphism classes of bounded odd Fredholm modules over $A$ by $\operatorname{Fr}(A)$. The set $\operatorname{Fr}(A)$ forms an Abelian semigroup under the operation

$$
\left(\pi_{0}, F_{0}\right)+\left(\pi_{1}, F_{1}\right):=\left(\pi_{0} \oplus \pi_{1}, F_{0} \oplus F_{1}\right) .
$$

Following Ref. 11, we define the odd analytic $K$-homology $K^{1}(A)$ as the set of homotopy classes of $\operatorname{Fr}(A)$. The homotopy class of a bounded odd Fredholm module $(\pi, F)$ will be denoted by $[(\pi, F)]$. The set $K^{1}(A)$ forms an Abelian group in the operation induced from $\operatorname{Fr}(A)$ by Theorem 2.1.23 of Ref. 11 .

If $\varphi: A \rightarrow B$ is a $*$-homomorphism we define $\varphi^{*}: K^{1}(B) \rightarrow K^{1}(A)$ by

$$
[(\pi, F)] \mapsto[(\pi \circ \varphi, F)] .
$$

The mapping $\varphi^{*}$ is a well defined group homomorphism. So we can view $K^{1}$ as a contravariant functor from the category of $C^{*}$-algebras to the category of Abelian groups. In addition to being homotopy invariant, the functor $K^{1}$ also satisfies half-exactness on semisplit sequences. That is, if $0 \rightarrow J \rightarrow A \rightarrow A / J \rightarrow 0$ is a short exact sequence of $C^{*}$-algebras admitting a completely positive splitting $A / J \rightarrow A$, the sequence of Abelian groups

$$
K^{1}(A / J) \rightarrow K^{1}(A) \rightarrow K^{1}(J)
$$

is exact at $K^{1}(A)$. To read more about exactness properties of $K$-homology, see Theorem 19.5.7 of Ref. 4.

An alternative description of $K^{1}$ is in terms of extensions. A short exact sequence of $C^{*}$-algebras $0 \rightarrow \mathcal{K}(\mathcal{H}) \rightarrow E \stackrel{q_{E}}{\rightarrow} A \rightarrow 0$ is called an extension of $A$ by $\mathcal{K}(\mathcal{H})$. We will sometimes abuse the notations and suppress the short exact sequence by identifying the $C^{*}$-algebra $E$ with the extension. In this setting the algebra $E$ should be viewed as the $C^{*}$-algebra generated by the quantized operators, $A$ as the $C^{*}$-algebra of symbols, and $q_{E}$ as the symbol mapping. Two extensions $E$ and $E^{\prime}$ are said to be unitarily equivalent if there exist a unitary $U \in \mathcal{B}(\mathcal{H})$ and a $*$-homomorphism $\psi: E \rightarrow E^{\prime}$, such that $\left.\psi\right|_{\mathcal{K}(\mathcal{H})}=\operatorname{Ad}(U)$ and $q_{E}=q_{E^{\prime}} \circ \psi$.

From an extension $E$ of $A$ by $\mathcal{K}(\mathcal{H})$ we can define a $*$-homomorphism $i_{E}: E \rightarrow \mathcal{B}(\mathcal{H})$ since $\mathcal{B}(\mathcal{H})$ is the multiplier algebra of $\mathcal{K}(\mathcal{H})$ and $\mathcal{K}(\mathcal{H})$ is an ideal in $E$, to read more about multiplier 
algebras, see Chapter 1 of Ref. 11. If $i_{E}$ is injective we may assume that $E \subseteq \mathcal{B}(\mathcal{H})$ and that the mapping $\mathcal{K}(\mathcal{H}) \rightarrow E$ is given by inclusion. The mapping $i_{E}$ does not need to be injective, for example, the mapping $i_{\tilde{\mathcal{T}}_{\mathrm{k}}}: \tilde{\mathcal{T}}_{\mathrm{k}} \rightarrow \mathcal{B}\left(\mathcal{L}_{\mathrm{k}}\right)$ associated with the extension in Eq. (3) is not injective.

The extension $E$ determines a $*$-homomorphism $\beta_{E}: A \rightarrow \mathcal{C}$ by $\beta_{E}(a):=\mathfrak{q}\left(i_{E}(\widetilde{a})\right)$, where $\widetilde{a} \in E$ is a preimage of $a$. The mapping $\beta_{E}$ is called the Busby mapping of $E$ and it determines the extension up to isomorphism. In fact, given a $*$-homomorphism $\beta: A \rightarrow \mathcal{C}$ we can define

$$
E_{\beta}:=\{a \oplus x \in A \oplus \mathcal{B}(\mathcal{H}): \beta(a)=\mathfrak{q}(x)\} .
$$

This gives an extension by letting $E_{\beta} \rightarrow A$ be the projection and $\mathcal{K}(\mathcal{H}) \rightarrow E_{\beta}$ be the embedding in the second coordinate. By Lemma 3.2.2 of Ref. 11 the unitary equivalence class of an extension is determined by its Busby mapping up to a unitary $U \in \mathcal{B}(\mathcal{H})$.

Let $B(A)$ denote the set of unitary equivalence classes of Busby mappings. The set $B(A)$ forms an Abelian semigroup in the operation,

$$
\left(\beta_{1}+\beta_{2}\right)(a):=\beta_{1}(a) \oplus \beta_{2}(a) \in \mathcal{C}(\mathcal{H} \oplus \mathcal{H}) \cong \mathcal{C}(\mathcal{H}) .
$$

A Busby mapping $\beta$ is called trivial if it is of the form $\beta=\mathfrak{q} \circ \lambda$ for a $*$-homomorphism $\lambda: A \rightarrow \mathcal{B}(\mathcal{H})$. We will denote the set of unitary equivalence classes of trivial Busby mappings by $B(A)_{0}$. The subset $B(A)_{0} \subseteq B(A)$ is a subsemigroup so we can define the semigroup quotient,

$$
\operatorname{Ext}(A):=B(A) / B(A)_{0} .
$$

We will denote the equivalence class of a Busby mapping $\beta$ by $[\beta]$. The drawback of extension theory is that $\operatorname{Ext}(A)$ is only a semigroup. There is a characterization of the $\operatorname{group} \operatorname{Ext}(A)^{-1}$ of invertible elements in $\operatorname{Ext}(A)$ which fits nicely together with $K$-homology. Theorem 3.2.9 of Ref. 11 states that an element $[\beta] \in \operatorname{Ext}(A)$ is invertible if and only if $[\beta]$ can be represented by a Busby mapping of the form $a \mapsto \mathfrak{q}(P \pi(a) P)$, where $P \in \mathcal{B}(\mathcal{H})$ satisfies that $\mathfrak{q}(P)$ is a projection and $\pi: A \rightarrow \mathcal{B}(\mathcal{H})$ is a representation, such that $[P, \pi(a)]$ is compact for any $a \in A$.

If $(\pi, F)$ is a bounded odd Fredholm module we consider the $*$-homomorphism $\beta^{F}: A \rightarrow \mathcal{C}$ defined by

$$
\beta^{F}(a):=\mathfrak{q}\left(P_{F} \pi(a) P_{F}\right), \quad \text { where } P_{F}:=\frac{1+F}{2} .
$$

By above the mapping $\gamma_{0}: \operatorname{Fr}(A) \rightarrow \operatorname{Ext}(A)^{-1},(\pi, F) \mapsto\left[\beta^{F}\right]$, is well defined. Lemma 3.3.8 of Ref. 11 states that the mapping $\gamma_{0}$ induces a mapping $\gamma: K^{1}(A) \rightarrow \operatorname{Ext}(A)^{-1}$ which by Theorem 3.3 .10 of Ref. 11 is an isomorphism. In particular, for nuclear $C^{*}$-algebras $A$ every element of $\operatorname{Ext}(A)$ is invertible and $K^{1}(A) \cong \operatorname{Ext}(A)$, see Corollary 15.8.4 of Ref. 4 .

Let us recall the definition of the index of an operator. If $T \in \mathcal{B}(\mathcal{H})$ is an operator such that $\operatorname{ker} T$ and $\operatorname{ker} T^{*}$ are finite dimensional, we say that $T$ is a Fredholm operator and define the index of $T$ as

$$
\operatorname{ind}(T):=\operatorname{dim} \operatorname{ker} T-\operatorname{dim} \operatorname{ker} T^{*} .
$$

The index is a homotopy invariant and invariant under compact perturbations. It also satisfies

$$
\operatorname{ind}\left(T_{1} T_{2}\right)=\operatorname{ind}\left(T_{1}\right)+\operatorname{ind}\left(T_{2}\right) .
$$

So the index induces a group homomorphism $\widetilde{\text { ind }}: \mathcal{C}^{-1} \rightarrow \mathbb{Z}$, where $\mathcal{C}^{-1}$ is the group of invertible elements in the Calkin algebra.

Applying this to $K$-theory we obtain that if $u \in \mathrm{GL}_{N}(A)$ and $(\pi, F)$ is a bounded odd Fredholm module, we can define $\operatorname{ind}_{F}(u)$ as the index of the operator,

$$
\left(P_{F} \otimes \mathrm{id}\right)(\pi \otimes \mathrm{id})(u): P_{F} \mathcal{H} \otimes \mathrm{C}^{N} \rightarrow P_{F} \mathcal{H} \otimes \mathrm{C}^{N} .
$$

Since the index is a homotopy invariant we can extend this construction to a pairing 


$$
K^{1}(A) \times K_{1}(A) \rightarrow \mathbb{Z} \quad \text { by } \quad[(\pi, F)] .[u]:=\operatorname{ind}_{F}(u) .
$$

So the pairing of $K$-theory and $K$-homology consists of the calculation of the index of the operator with symbol $u \in \mathrm{GL}_{N}(A)$ obtained by the quantization $a \mapsto P_{F} \pi(a) P_{F}$ of $A$ as defined in Eq. (A2).

${ }^{1}$ Atiyah, M. F., K-Theory, Lecture Notes by D. W. Anderson (W. A. Benjamin, New York, 1967).

${ }^{2}$ Avron, J. E., Seiler, R., and Simon, B., "Charge deficiency, charge transport and comparison of dimensions," Commun. Math. Phys. 159, 399 (1994).

${ }^{3}$ Berger, C. A. and Coburn, L. A., "Toeplitz operators on the Segal-Bargmann space," Trans. Am. Math. Soc. 301, 813 (1987).

${ }^{4}$ Blackadar, B., K-Theory for Operator Algebras, Mathematical Sciences Research Institute Publications Vol. 5, 2nd ed. (Cambridge University Press, Cambridge, 1998).

${ }^{5}$ Boutet de Monvel, L., "On the index of Toeplitz operators of several complex variables," Invent. Math. 50, 249 (1978).

${ }^{6}$ Brodzki, J., Mathai, V., Rosenberg, J., and Szabo, R. J., "D-branes, RR-fields, and duality on noncommutative manifolds," Commun. Math. Phys. 277, 643 (2008).

${ }^{7}$ Bruneau, V., Pushnitski, A., and Raikov, G., "Spectral shift function in strong magnetic fields," St. Petersburg Math. J. 16, 181 (2005).

${ }^{8}$ Douglas, R. G., Banach Algebra Techniques in the Theory of Toeplitz, Operators (AMS, Providence, 1972).

${ }^{9}$ Folland, G. B., Harmonic Analysis in Phase Space, Annals of Mathematics Studies Vol. 122 (Princeton University Press, Princeton, NJ, 1989).

${ }^{10}$ Guentner, E. and Higson, N., “A note on Toeplitz operators," Int. J. Math. 7, 501 (1996).

${ }^{11}$ Knudsen-Jensen, K. and Thomsen, K., Elements of KK-Theory (Birkhäuser, Boston, 1991).

${ }^{12}$ Melgaard, M. and Rozenblum, G., Stationary Partial Differential Equations, Handbook of Differential Equations Vol. 2, edited by Chipot, M. and Quittner, P. (Elsevier, Amsterdam, 2005), pp. 407-517.

${ }^{13}$ Murphy, G. J., C*-Algebras and Operator Theory (Academic, Boston, MA, 1990).

${ }^{14}$ Reis, R. M. G. and Szabo, R. J., "Geometric $K$-homology of flat $D$-branes," Commun. Math. Phys. 266, 71 (2006).

${ }^{15}$ Rosenberg, J. and Schochet, C., "The classification of extensions of $C^{*}$-algebras," Bull., New Ser., Am. Math. Soc. 4, 105 (1981).

${ }^{16}$ Rozenblum, G. and Sobolev, A. V., "Discrete spectrum distribution of the Landau operator perturbed by an expanding electric potential," e-print arXiv:0711.2158.

${ }^{17}$ Rozenblum, G. and Tashchiyan, G., "On the spectral properties of the perturbed Landau Hamiltonian," Commun. Partial Differ. Equ. 33, 1048 (2008).

${ }^{18}$ Venugopalkrishna, U., "Fredholm operators associated with strongly pseudoconvex domains in $\mathrm{C}^{n}$," J. Funct. Anal. 9, 349 (1972). 